\title{
SUBGROUP ALIGNMENT IN HYPERCOLOR THEORIES
}

\author{
John PRESKILL ${ }^{1}$ \\ Lyman Laboratory of Physics, Harvard University, Cambridge, Massachusetts 02138, USA
}

Received 7 July 1980

\begin{abstract}
To analyze the physical consequences of a dynamically broken theory of the weak interactions, we must know how the weak gauge group is aligned in an approximate flavor-symmetry group. For a large class of models, spectral-function sum rules enable us to determine this alignment explicitly. We work out the pattern of the electroweak symmetry breakdown for several sample models. Critical values of weak mixing angles are found at which the breakdown pattern changes discontinuously. We compute pseudo-Goldstone boson masses, and find that some models contain unusually light charged or colored pseudo-Goldstone bosons.
\end{abstract}

\section{Introduction}

The extremely successful Weinberg-Salam [1] model of the electroweak interactions is marred by one glaring imperfection-elementary scalar fields with negative mass squared are required to drive the breakdown of the electroweak gauge group. Such elementary scalars are distasteful for at least two reasons. First, we must adjust the bare scalar masses very delicately [2-4] to ensure that the mass scale of the electroweak breakdown $(300 \mathrm{GeV})$ is many orders of magnitude smaller than the grand unification [5] mass $\left(10^{15} \mathrm{GeV}\right)$ and the Planck mass $\left(10^{19} \mathrm{GeV}\right)$. Second, so many arbitrary parameters are needed to characterize the couplings of the scalars that we are reluctant to accept them as fundamental ingredients in the theory.

Dissatisfaction with the standard Weinberg-Salam model has spawned recent efforts to construct gauge theories of the electroweak interactions without elementary scalar fields. Weinberg [3] and Susskind [4] have proposed that the electroweak gauge group is actually broken dynamically by a postulated new strong interaction, rather than by elementary scalars. This new gauge interaction, which will be called "hypercolor" here, binds the Goldstone bosons which are eaten by the weak W and $Z$ bosons. Dimopoulos and Susskind [6], Eichten and Lane [7], and Weinberg [8] have noted that yet another gauge interaction, called "sideways", is required to generate the masses of quarks and leptons. The sideways gauge group must also be dynamically broken.

\footnotetext{
${ }^{1}$ Research supported in part by the National Science Foundation under grant number PHY77-22864 and the Harvard Society of Fellows.
} 
No one has yet constructed a fully realistic model based on these ideas. This failure is probably due to the tightly constrained nature of dynamically broken theories. These theories have few free parameters which can be adjusted to fit experiment. Lack of adjustability makes dynamically broken theories very appealing, but also makes it hard to find a theory which works.

To conduct a well-organized search for a realistic model, we must improve our still meager understanding of dynamical symmetry breaking. For example, consider an asymptotically free theory with gauge group $G$ and massless fermions in a reducible representation of $G$. Because the fermion representation is reducible, this theory respects a global flavor (chiral) symmetry group $G_{f}$, which commutes with $\mathrm{G}$. We would like to be able to answer the following questions:

(1) Is the gauge group $\mathrm{G}$ dynamically broken? If so, to what subgroup?

(2) Is the flavor-symmetry group $\mathrm{G}_{\mathrm{f}}$ dynamically broken? If so, to what subgroup?

But both (1) and (2) are difficult dynamical questions which we do not know how to answer in general.

In this paper, our attention will be focused instead on a somewhat more tractable question. If the flavor-symmetry group $G_{f}$ is dynamically broken to the subgroup $S_{f}$, then the theory has many degenerate vacua. The vacua can be parametrized by the coset space $G_{f} / S_{f}$; each vacuum corresponds to a particular orientation of $S_{f}$ in $G_{f}$. But if the $G_{f}$ symmetry is explicitly broken by a small perturbation, the degeneracy is lifted. The true vacuum of the theory then corresponds to the orientation of $S_{f}$ which minimizes the vacuum energy $[3,9]$. This orientation of $S_{f}$ is called the "orientation of the vacuum", or the "alignment of the vacuum". Thus, a third question which arises in dynamically broken theories is:

(3) If $G_{f}$ is dynamically broken to $S_{f}$, and explicitly broken by a small perturbation, what is the orientation of the vacuum?

The orientation of the vacuum, and its consequences in dynamically broken theories of the electroweak interactions, are the topics of this paper.

In electroweak gauge theories without elementary scalar fields, the $G_{f}$ flavor symmetry of the hypercolored fermions is dynamically broken to $S_{f}$ by the strong hypercolor interaction $[3,4], G_{f}$ is also explicitly, but weakly, broken by the sideways interaction [6,7] and by a weak gauge interaction with gauge group $G_{W} \subset G_{f} . \quad\left[G_{W}\right.$ contains the Weinberg-Salam $S U(2) \times U(1)$.] These flavorsymmetry-breaking perturbations determine the orientation of the vacuum.

The influence of the sideways interaction on the vacuum orientation has been considered elsewhere [10-12]. The sideways interaction can cause the vacuum to be oriented in such a way that $C P$ is spontaneously broken. It has been proposed [10-12] that the observed $C P$ violation is generated by this mechanism.

In this paper we consider the influence of the $G_{w}$ interaction on the vacuum orientation*. The problem of identifying the correct vacuum is equivalent to the

* The sideways interaction is ignored in this paper. Including it would not alter our results in any essential way. 
problem of finding the relative orientation of the subgroups $G_{w}$ and $S_{f}$ of $G_{f}$ which minimizes the vacuum energy. We call this the "subgroup alignment problem". The importance of the alignment of $G_{w}$ and $S_{f}$ was emphasized by Weinberg [3], who pointed out that this alignment determines the pattern of the $G_{w}$ breakdown and the spectrum of pseudo-Goldstone bosons. His general treatment of the subgroup alignment problem is reviewed in sects. 2 and 3.

Previous attempts to solve the subgroup alignment problem in particular cases have often been steeped in folklore ${ }^{\star}$. A popular notion is that gauge symmetries resist being broken, and that $G_{W}$ and $S_{f}$ will therefore line up so that the largest possible subgroup of $G_{w}$ survives. This point of view is sometimes expressed in a different way. The embedding of $S_{\mathrm{f}}$ in $\mathrm{G}_{\mathrm{f}}$ can be characterized by a fermion "condensate" [6]; according to the conventional wisdom, the condensate will form in the channel in which the lowest-order $\mathrm{G}_{\mathrm{w}}$ interaction is most attractive.

To solve the subgroup alignment problem properly, we must minimize an effective potential, the $G_{W}$ interaction contribution to the vacuum energy. Even though we assume that the weak $G_{w}$ interaction can be treated perturbatively, the problem is not trivial, because we cannot calculate the effective potential without solving the strong hypercolor interaction. Weinberg [3] observed that the symmetry properties of the hypercolor interaction, in particular the $S_{f}$ "isospin" symmetry, provide powerful constraints on the form of the effective potential. However, even in relatively simple cases we cannot find the minimum of the potential unless we know the signs of certain strong-interaction parameters which are not determined by $\mathrm{S}_{\mathrm{f}}$ symmetry alone.

The main conclusion ${ }^{\star \star}$ of this paper is that we can in many cases find the signs we need to know to minimize the effective potential, and that the results tend to confirm the conventional wisdom. Spectral function sum rules [15] provide the additional information we require. However, we can confirm the most attractive channel folklore only at the expense of introducing another element of folklore-we must assume that the signs of certain spectral integrals can be determined by saturating with low-lying resonances. Spectral function sum rules are reviewed in sect. 4 , and their relevance to the effective potential is explained in sect. 5 .

The ramifications of subgroup alignment are most easily appreciated in the context of specific models. The subgroup alignment problem is solved, and pseudoGoldstone boson masses are calculated, for a number of examples in sect. 6. These examples illustrate three important phenomena:

(i) Some hypercolor theories can be ruled out, because the dynamically determined pattern of $G_{w}$ breakdown is phenomenologically unacceptable.

(ii) If $G_{w}$ is not simple, the pattern of $G_{w}$ breakdown may depend on the relative strength of the different $\mathbf{G}_{\mathbf{W}}$ gauge couplings. There are critical values of weak mixing angles at which phase transitions occur.

\footnotetext{
* See, for example, [6] and [13].

$\star \star$ As this work was being completed, I learned that Peskin [14] has also analyzed subgroup alignment in hypercolor models, and has reached very similar conclusions.
} 
(iii) There may be pseudo-Goldstone bosons which receive mass not in lowest order in the $\mathrm{G}_{\mathrm{w}}$ interaction, but in higher order. Hence, some charged or colored pseudo-Goldstone bosons may be considerably lighter than we would naively expect [7].

In this paper, we do not address questions (1) and (2) stated above, but the most attractive channel folklore has been applied to these questions also. Several authors [16] have speculated recently on the pattern of breakdown of gauge and global symmetries in a confining gauge theory with non-real fermion representation content. Lacking the basis for a detailed dynamical analysis, they have assumed that a fermion condensate occurs in the channel which is most attractive in lowest order in the gauge coupling. Of course, the gauge interaction must be strong to bind Goldstone bosons, so lowest-order perturbation theory is completely untrustworthy when dynamical symmetry breaking occurs. We would feel less uncomfortable about applying the most attractive channel condition here if there were some justification for it which goes beyond perturbation theory. No such justification is known.

This paper might be regarded as a modest attempt to justify the most attractive channel condition in a relatively simple context. By assumption, it is a good approximation to treat the $G_{w}$ interaction to lowest order, but the strong hypercolor interaction must be summed to all orders. Taking into account the strong corrections, we find, in the cases we can explicitly analyze, that the subgroups $\mathbf{G}_{\mathbf{w}}$ and $S_{f}$ tend to align so that the fermion condensate occurs in the channel in which the lowest-order $\mathrm{G}_{\mathrm{w}}$ interaction is most attractive.

\section{The subgroup alignment problem}

In this section and sect. 3 we review the general formulation of the subgroup alignment problem given by Weinberg [3].

We consider a gauge theory with massless fermions and no elementary scalars. The gauge group is $G_{H} \times G_{W} \cdot G_{H}$ is a simple group, the hypercolor group. The associated running coupling constant becomes strong at a mass scale near $1 \mathrm{TeV}$. $\mathrm{G}_{\mathrm{w}}$ is not necessarily simple. All the $\mathrm{G}_{\mathrm{w}}$ gauge couplings are weak at $1 \mathrm{TeV}$.

It is convenient to choose all fermions to be two-component left-handed spinors. The fermions transform as a representation $\mathscr{D}$ of $G_{H}$ which in general is reducible; we have

$$
\mathscr{D}=\prod_{\rho} n_{\rho} \mathscr{Q D}^{(\rho)}
$$

That is, the irreducible representation $\mathscr{D}^{(\rho)}$ is repeated $n_{\rho}$ times. The fermions may be denoted $\psi_{r i}^{(\rho)}$, where $\rho$ identifies the irreducible representation of $\mathrm{G}_{\mathrm{H}}$ according to which $\psi^{(\rho)}$ transforms, $i$ is the index on which $\mathrm{G}_{\mathrm{H}}$ acts, and $r=1, \ldots, n_{\rho}$ labels the different flavors of fermions which transform as $\mathscr{Q D}^{(\rho)}$. The group $\mathrm{G}_{\mathrm{w}}$ acts on the index $r$. 
The lagrangian of this theory is

$$
\begin{aligned}
\mathcal{L}= & -\frac{1}{4} F_{\mathrm{H} \mu \nu}^{a} F_{\mathrm{H}}^{\mu \nu a}-\frac{1}{4} F_{\mathrm{W}_{\mu \nu}}^{\alpha} F_{\mathrm{W}}^{\mu \nu \alpha}+\bar{\psi}_{r i}^{(\rho)}\left(i \gamma^{\mu} \partial_{\mu}\right) \psi_{r i}^{(\rho)} \\
& +\bar{\psi}_{r i}^{(\rho)} \gamma^{\mu}\left(g_{\mathrm{H}} t_{i j}^{(\rho) a} A_{\mathrm{H}_{\mu}}^{a}\right) \psi_{r j}^{(\rho)} \\
& +\bar{\psi}_{r i}^{(\rho)} \gamma^{\mu}\left(e_{\alpha} \theta_{r r^{\prime}}^{(\rho) \alpha} A_{\mathrm{W}_{\mu}}^{\alpha}\right) \psi_{r^{\prime} i}^{(\rho)}
\end{aligned}
$$

(all indices summed). $A_{\mathrm{H}}$ and $A_{\mathrm{W}}$ are the $\mathrm{G}_{\mathrm{H}}$ and $\mathrm{G}_{\mathrm{W}}$ gauge fields; $F_{\mathrm{H}}$ and $F_{\mathrm{W}}$ are the corresponding covariant curls. The $t^{(\rho)}$ 's are the $\mathrm{G}_{\mathrm{H}}$ generators in the representation $\mathscr{D}^{(\rho)}$, and the $\theta^{(\rho)}$ 's are the $\mathrm{G}_{\mathrm{W}}$ generators. The gauge couplings are $g_{\mathrm{H}}$ and $e_{\alpha}$.

In the limit $e \rightarrow 0$, this lagrangian is invariant under transformations of the form

$$
\psi_{r i}^{(\rho)} \rightarrow U_{r r^{\prime}}^{(\rho)} \psi_{r^{\prime}}^{(\rho)},
$$

where $U^{(\rho)}$ is a unitary $n_{\rho} \times n_{\rho}$ matrix. Because there is one $\mathrm{U}(1)_{\mathrm{A}}$ transformation which has $a_{\mathbf{H}_{\mathbf{H}}}$ anomaly [17], the global flavor-symmetry group of this theory is

$$
\mathrm{G}_{\mathrm{f}}=\prod_{\rho} \mathrm{U}\left(n_{\rho}\right) / \mathrm{U}_{\mathrm{A}}(1)
$$

in the $e=0$ limit. The weak gauge group $\mathrm{G}_{\mathrm{W}}$ is a subgroup of $\mathrm{G}_{\mathrm{f}}$.

When the hypercolor interaction becomes strong, $G_{f}$ is spontaneously broken to an "isospin" subgroup $\mathrm{S}_{\mathrm{f}}$. (If $e$ is not zero but small, the $\mathrm{G}_{\mathrm{w}}$ interaction has little influence on the strong hypercolor dynamics, and we do not expect the pattern of $G_{f}$ breaking to be altered.) Although it is generally believed that chiral symmetries break in confining theories [18], we have no reliable way of computing $S_{\mathrm{f}}$. The only theory for which we have experimental information is QCD; there, SU(3) $\times \mathrm{SU}(3)$ chiral symmetry appears to break to the maximal diagonal isospin subgroup SU(3), but we do not know if this represents a general phenomenon or is merely one of several natural possibilities [19]. For now, we allow $S_{\mathrm{f}}$ to be an arbitrary subgroup of $\mathrm{G}_{\mathrm{f}}$. Special cases will be discussed in sects. 5 and 6 .

In the $e=0$ limit, $\mathrm{G}_{\mathrm{f}}$ symmetry is exact, and the vacuum is highly degenerate; the vacua are parametrized by the coset space $G_{t} / S_{\mathrm{f}}$. If $e$ is non-zero but small, $\mathrm{G}_{\mathrm{w}}$-boson exchange generates a weak $\mathrm{G}_{\mathrm{f}}$-breaking perturbation $\mathcal{K}^{\prime}$. This perturbation lifts the degeneracy and picks out the true vacuum. As Dashen [9] was the first to observe, a perturbation expansion in $\mathcal{H}^{\prime}$ must be performed about the correct vacuum to avoid paradoxial results.

The correct vacuum can be identified by minimizing an effective potential (the vacuum energy), given to lowest order in $\mathcal{H}^{\prime}$ by

$$
V(g)=\left\langle 0\left|U(g)^{-1} \mathcal{H}^{\prime} U(g)\right| 0\right\rangle=\left\langle 0, g\left|\mathcal{K}^{\prime}\right| 0, g\right\rangle,
$$




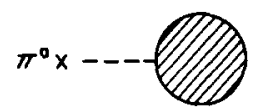

Fig. 1. A Goldstone boson tadpole.

where $U(g)$ represents $G_{f}$ in Hilbert space. If the vacuum $|0\rangle$ is left invariant by $\mathrm{S}_{\mathrm{f}} \subset \mathrm{G}_{\mathrm{f}}$, then $U(g)|0\rangle=|0, g\rangle$ is left invariant by the equivalent chirally rotated subgroup $\mathrm{gS}_{\mathrm{f}} \mathrm{g}^{-1}$.

We can also interpret eq. (2.5) by regarding the vacuum $|0\rangle$ as fixed, so that the chiral rotation is applied to the perturbation $\mathcal{K}^{\prime}$. Then we minimize $V(g)$ by finding the chiral perturbation $\mathcal{K}^{\prime}(g)=U(g)^{-1} \mathcal{K}^{\prime} U(g)$ which gives the lowest contribution to the energy of the $S_{\mathrm{f}}$-invariant vacuum. If we express $U\left(g g^{\prime}\right)$ in terms of the chiral charges $Q_{a}, U\left(g g^{\prime}\right)=U(g) \exp \left[i \omega_{a}\left(g^{\prime}\right) Q_{a}\right]$, the condition satisfied by $\mathcal{H}^{\prime}(g)$ at the minimum is

$$
\begin{gathered}
\left.\frac{\mathrm{d} V}{\mathrm{~d} \omega_{a}}\right|_{\omega=0}=i\left\langle 0\left|\left[\mathcal{H}^{\prime}(g), Q_{a}\right]\right| 0\right\rangle=0, \\
\left.\frac{\mathrm{d}^{2} V}{\mathrm{~d} \omega_{a} \mathrm{~d} \omega_{b}}\right|_{\omega=0}=-\left\langle 0\left|\left[\left[\mathcal{H}^{\prime}(g), Q_{a}\right], Q_{b}\right]\right| 0\right\rangle \geqslant 0 .
\end{gathered}
$$

( $Q$ may not be defined if the associated symmetry is spontaneously broken, but the commutator of $Q_{a}$ with a local operator can still be defined.)

These equations have a straightforward current algebra interpretation. Charges $Q_{a}$ which do not annihilate the vacuum couple to Goldstone bosons. Eq. (2.6) says that Goldstone boson tadpoles vanish (fig. 1). If the perturbation $\mathcal{H}^{\prime}(g)$ has a non-vanishing matrix element to create a Goldstone boson, $\left\langle\pi^{a}\left|\mathcal{H}^{\prime}\right| 0\right\rangle \neq 0$, the Goldstone bosons can be produced spontaneously, and the vacuum is unstable. Eq. (2.7) guarantees that the Goldstone boson mass matrix [20],

$$
m_{a b}^{2}=-\frac{1}{F_{a} F_{b}}\left\langle 0\left|\left[\left[\mathcal{H}^{\prime}(g), Q_{a}\right], Q_{b}\right]\right| 0\right\rangle,
$$

has no negative eigenvalues (fig. 2). A Goldstone boson tachyon also signals an instability.

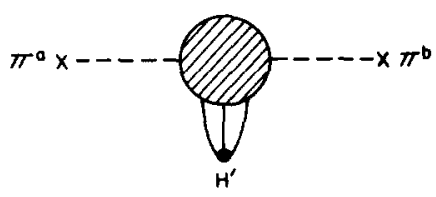

Fig. 2. Lowest-order contribution to the PGB mass matrix $\boldsymbol{m}_{a b}^{2}$. 
Because the symmetry group $G_{f}$ is compact, $V(g)$ always has a global minimum and a global maximum. The true vacuum corresponds to the global minimum, but there may also be metastable false vacua corresponding to local minima which are not global minima.

Because $\mathcal{H}^{\prime}$ is generated by the exchange of $G_{W}$ gauge bosons, the $G_{f}$ transformation $U(g)$ has the effect of changing the embedding of $\mathrm{G}_{\mathrm{W}}$ in $\mathrm{G}_{\mathrm{f}}$ :

$$
\mathrm{G}_{\mathrm{w}} \rightarrow g^{-1} \mathrm{G}_{\mathrm{w}} g \text {. }
$$

Minimizing $V(g)$ determines the relative orientation of the subgroups $S_{\mathrm{f}}$ and $\mathrm{G}_{\mathrm{W}}$ in $G_{\mathrm{f}}$. Hence, the problem of finding the minimum of the effective potential when a subgroup of $G_{f}$ is gauged is called the "subgroup alignment problem" [3].

We must know how the subgroups are aligned to determine how $G_{w}$ breaks, to classify Goldstone bosons, and to calculate their masses. The general classification of Goldstone bosons in a theory like the one defined by eq. (2.2) was carried out by Weinberg [3]. He observed that the global symmetry group of the $G_{w}$ interactions may be larger than the local group $G_{W}$. Call this global group $G_{f}^{\prime}$. When $G_{f}$ breaks down to $S_{\mathrm{f}}$, the resulting Goldstone bosons are in one-to-one correspondence with an orthonormal set of broken currents. The Goldstone bosons fall into three classes:

(i) Fictitious. If the corresponding current is a linear combination of $\mathbf{G}_{\mathbf{W}}$ currents and $S_{f}$ currents, then the Goldstone boson is eaten by a $G_{w}$ gauge boson.

(ii) Exact. If the corresponding current is a linear combination of $\mathrm{G}_{\mathrm{f}}^{\prime}$ currents which are not $G_{W}$ currents and $S_{f}$ currents, then the Goldstone boson is exactly massless.

(iii) Pseudo. If the corresponding current is not a linear combination of $\mathrm{G}_{\mathrm{f}}^{\prime}$ currents and $S_{f}$ currents, then the Goldstone boson receives mass from the perturbation $\mathcal{K}^{\prime}$. It is called a pseudo-Goldstone boson (PGB).

We must solve the subgroup alignment problem before we can classify the Goldstone bosons.

The $G_{W}$ vector bosons acquire masses by eating fictitious Goldstone bosons. If the $\mathrm{G}_{\mathrm{W}}$ currents $J_{\mu}^{\alpha}=\bar{\psi}_{r i}^{(\rho)} \gamma_{\mu} \theta_{r r^{\prime}}^{(\rho) \alpha} \psi_{r^{i}}^{(\rho)}$ couple to Goldstone bosons,

$$
\left\langle 0\left|J_{\mu}^{\alpha}\right| \pi^{A}\right\rangle=i k_{\mu} F^{\alpha A},
$$

then the polarization tensor $\pi_{\alpha \beta}$ defined by

$$
e_{\alpha} e_{\beta} \int \mathrm{d}^{4} x \mathrm{e}^{i k x}\left\langle 0\left|\mathrm{~T}\left[J_{\mu}^{\alpha}(x) J_{\nu}^{\beta}(0)\right]\right| 0\right\rangle=i\left(\eta_{\mu \nu} k^{2}-k_{\mu} k_{\nu}\right) \pi_{\alpha \beta}\left(k^{2}\right)
$$

has a pole at $k^{2}=0$. The residue of this pole,

$$
\mu_{\alpha \beta}^{2}=e_{\alpha} e_{\beta} \sum_{A} F^{\alpha A} F^{\beta A},
$$


is the $\mathrm{G}_{\mathrm{W}}$ vector boson mass matrix [21]. The $F^{\alpha A}$ 's and therefore also $\mu^{2}$, depend on the relative orientation of $G_{W}$ and $S_{f}$. We must solve the subgroup alignment problem to determine the pattern of $G_{W}$ breakdown.

Because the Goldstone bosons form $S_{\mathrm{f}}$ isospin multiplets, the $F^{\alpha A}$ 's obey relations which are consequences of $S_{f}$ invariance. Weinberg [3] and Susskind [4] have emphasized that $S_{f}$ invariance can require the weak vector boson masses given by (2.12) to obey the relation

$$
\mu_{\mathrm{w}}^{2} / \mu_{\mathrm{Z}}^{2}=\cos ^{2} \theta_{\mathrm{w}} .
$$

This relation also holds in the Weinberg-Salam model with an elementary scalar doublet [1], and is known to be well satisfied.

\section{The effective potential}

Having established the importance of determining the alignment of subgroups, we now turn to the problem of actually constructing and minimizing the effective potential $V(g)$. In this section we show that, by exploiting the $S_{\mathrm{f}}$ invariance of the vacuum, we can write down a compact expression for $V(g)$ in terms of a few unknown matrix elements.

Because the $G_{w}$ interaction is weak at the mass scale $(1 \mathrm{TeV})$ at which hypercolor becomes strong, we may compute the effective potential in a perturbation series in $e$. To lowest order in $e$, the $\mathrm{G}_{\mathrm{f}}$-breaking perturbation $\mathcal{H}^{\prime}$ is

$$
\mathcal{H}^{\prime}=\left(-\frac{1}{2} i\right) \int \mathrm{d}^{4} x \Delta^{\mu \nu}(x) e_{\alpha}^{2} \mathrm{~T}\left[J_{\mu}^{\alpha}(x) J_{\nu}^{\alpha}(0)\right],
$$

where $\Delta^{\mu \nu}$ is the gauge boson propagator and $J_{\mu}^{\alpha}$ is a $\mathrm{G}_{\mathrm{W}}$ current.

In order to clearly exhibit the way the effective potential varies under $G_{f}$ rotations, it is convenient to express the $G_{w}$ currents in terms of a standard basis of $G_{f}$ currents. We denote this basis by

$$
J_{\mu}^{A}=\bar{\psi}_{r i}^{(\rho)} \gamma_{\mu} \lambda_{r r^{\prime}}^{(\rho) A} \psi_{r^{i}}^{(\rho)}
$$

where the $\lambda^{A}$ 's are a basis for the generators of $\mathrm{G}_{\mathrm{f}}$, normalized so that

$$
\operatorname{Tr} \lambda^{A} \lambda^{B}=\delta^{A B} .
$$

Under $G_{\mathrm{f}}$ transformations, the currents $J^{A}$ transform as the adjoint representation of $G_{f}$; that is

$$
J^{A}(g)=U^{-1}(g) J^{A} U(g)=R^{A B}(g) J^{B},
$$

where $R(g)$ is the adjoint representation. 


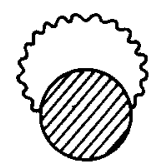

Fig. 3. Lowest-order $G_{W}$ contribution to the vacuum energy.

The $G_{W}$ currents are linear combinations of $G_{f}$ currents,

$$
e_{\alpha} J_{\mu}^{\alpha}=e_{\alpha A} J_{\mu}^{A}, \quad(\alpha \text { not summed })
$$

and we can combine (2.5) with (3.1), (3.4) and (3.5) to obtain (fig. 3)

$$
V(g)=e_{\alpha A} e_{\alpha B} R^{A C}(g) R^{B D}(g) I^{C D},
$$

where

$$
I^{C D}=\left(-\frac{1}{2} i\right) \int \mathrm{d}^{4} x \Delta^{\mu \nu}(x)\left\langle 0\left|\mathrm{~T}\left[J_{\mu}^{C}(x) J_{\nu}^{D}(0)\right]\right| 0\right\rangle
$$

This expression for $V(g)$ was derived by Weinberg [3].

In (3.6), we have expressed the effective potential in terms of group-theoretic factors, $G_{w}$ gauge couplings, and the $G_{f}$ tensor $I^{A B}$. Because the vacuum $|0\rangle$ is $S_{f}$ invariant, we see from (3.7) that $I^{A B}$ is also $S_{f}$ invariant. We can decompose the adjoint representation $\mathrm{Ad}\left(\mathrm{G}_{\mathrm{f}}\right)$ into irreducible representations of $\mathrm{S}_{\mathrm{f}}$. The tensor $I^{A B}$ can then be expressed in terms of as many unknown constants as there are $S_{f}$ singlets in $\operatorname{Ad}\left(G_{f}\right) \times \operatorname{Ad}\left(G_{f}\right)^{\star}$.

For example, if the currents $J^{A}, A=1,2, \ldots, n_{\rho}$, are in the (real) irreducible representation $\mathscr{D}^{(\rho)}$ of $S_{\mathrm{f}}$, then, by Schur's lemma,

$$
I^{A B}=\Delta_{\rho} \delta^{A B}, \quad A, B=1,2, \ldots, n_{\rho} .
$$

However, we cannot determine the constant $\Delta_{\rho}$ without solving the strong hypercolor interaction.

\section{Spectral function sum rules}

To minimize the effective potential given by (3.6), we must know more about the tensor $I^{A B}$. In sect. 5, we will argue that the additional information we seek can of ten be extracted from spectral function sum rules, which are therefore the subject of the present section.

\footnotetext{
* The number of independent invariants can often be further reduced by invoking additional
} symmetries, such as CPT and parity. 
Spectral function sum rules (SFSR's) were first derived by Weinberg [15] for the case of spontaneously broken $\mathrm{SU}(2) \times \mathrm{SU}(2)$ chiral symmetry. Wilson [22] and Bernard, Duncan, Lo Secco, and Weinberg [23] put the derivation on a more secure footing by invoking the operator product expansion ${ }^{\star}$. Here we will review the derivation of Bernard et al., which was carried out in the context of spontaneously broken $\mathrm{SU}(N) \times \mathrm{SU}(N)$ chiral symmetry, and indicate how it can be generalized to arbitrary $G_{f}$ breakdown patterns.

The starting point of all derivations of the SFSR's is the Lehmann-Källen spectral representation for the (time-ordered) product of two currents. If we define the spin-one and spin-zero spectral functions associated with a pair of currents $J_{\mu}^{A}$ and $J_{v}^{B}$ by

$$
\begin{aligned}
& (2 \pi)^{3} \sum_{\substack{n \\
\text { (spin 1) }}}\left\langle 0\left|J_{\mu}^{A}(0)\right| n\right\rangle\left\langle n\left|J_{\nu}^{B}(0)\right| 0\right\rangle \delta^{4}\left(k-k_{n}\right)=-\left(\eta_{\mu \nu}-\frac{k_{\mu} k_{\nu}}{k^{2}}\right) \rho_{A B}^{(1)}\left(k^{2}\right), \\
& (2 \pi)^{3} \sum_{\substack{n \\
\text { (spin 0) }}}^{\sum_{\text {spin }}}\left\langle 0\left|J_{\mu}^{A}(0)\right| n\right\rangle\left\langle n\left|J_{\nu}^{B}(0)\right| 0\right\rangle \delta^{4}\left(k-k_{n}\right)=k_{\mu} k_{\nu} \rho_{A B}^{(0)}\left(k^{2}\right),
\end{aligned}
$$

then we find by inserting a complete set of intermediate states that

$$
\begin{aligned}
\left\langle 0\left|\mathrm{~T}\left[J_{\mu}^{A}(x) J_{\nu}^{B}(0)\right]\right| 0\right\rangle= & \int_{0}^{\infty} \mathrm{d} \mu^{2} \int \frac{\mathrm{d}^{4} k}{(2 \pi)^{4}} \mathrm{e}^{-i k x} \frac{-i}{k^{2}-\mu^{2}+i \varepsilon} \\
& \times\left[\rho_{A B}^{(1)}\left(\mu^{2}\right)\left(\eta_{\mu \nu}-\frac{k_{\mu} k_{\nu}}{\mu^{2}}\right)-\rho_{A B}^{(0)}\left(\mu^{2}\right) k_{\mu} k_{\nu}\right] .
\end{aligned}
$$

SFSR's are derived by considering the behavior of both sides of eq. (4.3) in the short-distance limit. It is most convenient to expand the Fourier transform in powers of $1 / k^{2}$. We have

$$
\begin{aligned}
\int \mathrm{d}^{4} x \mathrm{e}^{i k x}\left\langle 0\left|\mathrm{~T}\left[J_{\mu}^{A}(x) J_{\nu}^{B}(0)\right]\right| 0\right\rangle= & i \frac{k_{\mu} k_{\nu}}{k^{2}} \int_{0}^{\infty} \mathrm{d} \mu^{2}\left[\frac{\rho_{A B}^{(1)}}{\mu^{2}}+\rho_{A B}^{(0)}\right] \\
& -i \frac{\eta_{\mu \nu}}{k^{2}} \int_{0}^{\infty} \mathrm{d} \mu^{2}\left[\rho_{A B}^{(1)}\right] \\
& +i \frac{k_{\mu} k_{\nu}}{\left(k^{2}\right)^{2}} \int_{0}^{\infty} \mathrm{d} \mu^{2}\left[\rho_{A B}^{(1)}+\mu^{2} \rho_{A B}^{(0)}\right]+\cdots
\end{aligned}
$$

* See also Hagiwara and Mohapatra [29]. 
Ordinarily the left-hand side of eq. (4.4) is expected to behave like $O\left(k^{2}\right)$ for large $k^{2}$. Then the expansion on the right-hand side cannot be valid; presumably it fails because the coefficients are divergent. However, if we construct a linear combination of current products which is softer than $1 / k^{2}$ for large $k^{2}$, then the first few terms in the expansion in eq. (4.4) must vanish, and the corresponding linear combinations of spectral functions satisfy the relations

$$
\begin{array}{r}
\int_{0}^{\infty} \mathrm{d} s\left[\rho^{(1)} / s+\rho^{(0)}\right]=0, \\
\int_{0}^{\infty} \mathrm{d} s \rho^{(1)}=0, \\
\int_{0}^{\infty} \mathrm{d} s s \rho^{(0)}=0 .
\end{array}
$$

These are the SFSR's.

To find linear combinations of current products with soft high-momentum behavior, we use Wilson's operator product expansion [22]. Bernard et al. [23] have shown that, if $G_{f}$ chiral symmetry is a symmetry of the lagrangian, then it is respected by the Wilson coefficient functions, whether or not $G_{f}$ is spontaneously broken. The $G_{f}$ symmetry of the coefficient functions enables us to find spectral functions which obey SFSR's.

As an example, consider the familiar case in which $\mathrm{G}_{\mathrm{f}}=\mathrm{SU}(N)_{\mathrm{L}} \times \mathrm{SU}(N)_{\mathrm{R}}$ and $\mathrm{S}_{\mathrm{f}}=\mathrm{SU}(N)_{\mathrm{V}}$, the case realized by QCD. A SFSR can be derived by studying the high-momentum limit of

$$
M_{\mu \nu}^{A B}(k)=\int \mathrm{d}^{4} x \mathrm{e}^{i k x}\left\langle 0\left|\mathrm{~T}\left[J_{L \mu}^{A}(x) J_{R \nu}^{B}(0)\right]\right| 0\right\rangle
$$

which transforms as (Ad, Ad) under $\operatorname{SU}(N)_{\mathrm{L}} \times \mathrm{SU}(N)_{\mathrm{R}}$, where Ad is the adjoint representation of $\mathrm{SU}(N)$. The asymptotic behavior of $M_{\mu \nu}^{A B}(k)$ is determined by the lowest-dimension operator in the Wilson expansion of $J_{L_{\mu}}^{A} J_{R_{\nu}}^{B}$ which has a vacuum expectation value. This operator must be Lorentz invariant, gauge invariant, and $S_{f}$ invariant, and, because the Wilson coefficient functions respect the $G_{f}$ symmetry, it must transform as (Ad, Ad) under $G_{f}$. The lowest-dimension operator meeting these criteria is a four-fermion operator of dimension (mass) ${ }^{6}$. Therefore, $M^{A B} \sim$ $\left(k^{2}\right)^{-2}\left(\log k^{2}\right)^{P}$, and the SFSR's (4.5) hold for the spectral function $\rho_{\mathrm{LR}}$.

It is customary to express these SFSR's in terms of the vector and axial vector spectral functions $\rho_{\mathrm{V}}$ and $\rho_{\mathrm{A}}$. Invariance under parity and $\mathrm{SU}(N)$ isospin implies

$$
\begin{aligned}
& \left\langle 0\left|V_{\mu}^{A}(x) V_{\nu}^{B}(0)\right| 0\right\rangle \propto \delta^{A B}, \\
& \left\langle 0\left|A_{\mu}^{A}(x) A_{\nu}^{B}(0)\right| 0\right\rangle \propto \delta^{A B}, \\
& \left\langle 0\left|V_{\mu}^{A}(x) A_{\nu}^{B}(0)\right| 0\right\rangle=0,
\end{aligned}
$$


so that

$$
M_{\mu \nu}^{A B}(k)=\frac{1}{4} \delta^{A B} \int \mathrm{d}^{4} x \mathrm{e}^{i k x}\left\langle 0\left|\mathrm{~T}\left[V_{\mu}^{A}(x) V_{\mu}^{A}(0)-A_{\mu}^{A}(x) A_{\nu}^{A}(0)\right]\right| 0\right\rangle
$$

The axial vector currents couple to the Goldstone bosons,

$$
\left\langle 0\left|A_{\mu}^{B}\right| \pi^{A}\right\rangle=i k_{\mu} F \delta^{A B},
$$

and therefore,

$$
\rho_{\mathrm{A}}^{(0)}\left(\mu^{2}\right)=F^{2} \delta\left(\mu^{2}\right) .
$$

Now, combining (4.5), (4.8) and (4.10), we find

$$
\begin{gathered}
\int \frac{\mathrm{d} s}{s}\left[\rho_{\mathrm{V}}(s)-\rho_{\mathrm{A}}(s)\right]=F^{2}, \\
\int \mathrm{d} s\left[\rho_{\mathrm{V}}(s)-\rho_{\mathrm{A}}(s)\right]=0 .
\end{gathered}
$$

These are Weinberg's SFSR's [15], which are exact relations in the chiral limit.

Next, consider the case of $N$ flavors of (left-handed) fermions. $\mathrm{G}_{f}$ is $\mathrm{SU}(N)$ and $S_{\mathrm{f}}$ is a subgroup of $G_{\mathrm{f}}$. The $G_{\mathrm{f}}$ currents transform as the adjoint representation $\operatorname{Ad}\left(G_{f}\right)$, and the product of two $G_{f}$ currents transforms as $\operatorname{Ad}\left(G_{f}\right) \times \operatorname{Ad}\left(G_{f}\right)=1+$ $\operatorname{Ad}\left(G_{f}\right)+\operatorname{Ad}\left(G_{f}\right)+\cdots$. Here $\cdots$ represents other non-trivial representations, all of which, of course, have $N$-ality zero. Unless $N=2$, the only operators which are gauge invariant, Lorentz invariant, have $N$-ality zero and dimensionality less than six are $G_{f}$-singlet operators which contain no fermion fields. $\left(\bar{\psi} \gamma^{\mu} D_{\mu} \psi\right.$ can be eliminated by the equations of motion.) Therefore, there is an SFSR associated with each $S_{f}$ singlet linear combination of current products which does not contain the $\mathrm{G}_{\mathrm{f}}$ singlet. The number of independent SFSR's is one less than the number of $S_{\mathrm{f}}$ singlets in $\operatorname{Ad}\left(G_{\mathrm{f}}\right) \times \operatorname{Ad}\left(G_{\mathrm{f}}\right)$. (Although the above argument breaks down if $N=2$, the SFSR's hold in that case also.)

For each multiplet of broken currents in the representation $D$ of $S_{f}$, the corresponding combination $\left(\rho_{\mathrm{Ad}}-\rho_{\mathrm{D}}\right)$ obeys SFSR's, where $\rho_{\mathrm{Ad}}$ is the spectral function associated with an irreducible representation of unbroken currents. We have the relations

$$
\begin{gathered}
\int \frac{\mathrm{d} s}{s}\left[\rho_{\mathrm{Ad}}(s)-\rho_{\mathrm{D}}(s)\right]=F_{\mathrm{D}}^{2}>0, \\
\int \mathrm{d} s\left[\rho_{\mathrm{Ad}}(s)-\rho_{\mathrm{D}}(s)\right]=0 .
\end{gathered}
$$


If $\mathrm{S}_{\mathrm{f}}$ is trivial, SFSR's are satisfied by $\rho_{\mathrm{D}_{1}}-\rho_{\mathrm{D}_{2}}$, with $F_{\mathrm{D}_{2}}^{2}-F_{\mathrm{D}_{1}}^{2}$ on the right-hand side of the first sum rule.

If the equivalent but distinct representations $D$ and $D^{\prime}$ of $S_{f}$ occur in $\operatorname{Ad}\left(G_{f}\right)$, then the corresponding spectral function $\rho_{\mathrm{DD}}$, obeys the SFSR's

$$
\begin{aligned}
& \int \frac{\mathrm{d} s}{s} \rho_{\mathrm{DD}^{\prime}}(s)=F_{\mathrm{DD}^{\prime}}^{2}, \\
& \int \mathrm{d} s \rho_{\mathrm{DD}^{\prime}}(s)=0 .
\end{aligned}
$$

In general, the $\mathrm{D}$ and $\mathrm{D}^{\prime}$ Goldstone boson multiplets can mix, and $F_{\mathrm{DD}^{\prime}}^{2}$ need not vanish. However, $\mathrm{D}$ and $\mathrm{D}^{\prime}$ can always be chosen such that there is no mixing, and $F_{\mathrm{DD}^{\prime}}^{2}=0$.

If $\mathrm{S}_{\mathrm{f}}$ is not simple, then we also have

$$
\begin{gathered}
\int \frac{\mathrm{d} s}{s}\left(\rho_{\mathrm{Ad}}-\rho_{\mathrm{Ad}^{\prime}}\right)=0, \\
\int \mathrm{d} s\left(\rho_{\mathrm{Ad}}-\rho_{\mathrm{Ad}^{\prime}}\right)=0,
\end{gathered}
$$

where $\rho_{\mathrm{Ad}}$ and $\rho_{\mathrm{Ad}}$ are spectral functions associated with unbroken currents in different factors of $S_{\mathbf{r}}$.

It is now clear that SFSR's can be derived for arbitrary $G_{f}$ and $S_{f} \subset G_{f}$. The total number of independent SFSR's is the number of $S_{f}$ singlets less the number of $G_{f}$ singlets contained in $\operatorname{Ad}\left(G_{f}\right) \times \operatorname{Ad}\left(G_{f}\right)$.

The SFSR's (4.12)-(4.14) hold in the limit of exact $G_{f}$ symmetry. In the theories we are interested in, $G_{f}$ is explicitly broken by the $G_{w}$ interaction (and by the sideways interaction, too, in a realistic model). Nevertheless, we are justified in using (4.12)-(4.14) when calculating to lowest order in the $\mathrm{G}_{\mathrm{f}}$-breaking perturbation, as when we calculate the lowest-order $G_{w}$ contribution to the effective potential.

\section{Spectral functions and the effective potential}

In sect. 3 , the lowest-order $\mathrm{G}_{\mathrm{w}}$-boson exchange contribution to the effective potential was expressed in terms of an $\mathrm{S}_{\mathrm{f}}$-invariant tensor $I^{A B}$. The tensor $I^{A B}$ depends on several strong-interaction parameters. These parameters are spectral integrals. 
If we invoke the spectral representation (4.3), $I^{A B}$ becomes

$$
\begin{aligned}
I^{A B} & =-\frac{1}{2} i \int \mathrm{d}^{4} x \Delta^{\mu \nu}(x)\left\langle 0\left|\mathrm{~T}\left[J_{\mu}^{A}(x) J_{\nu}^{B}(0)\right]\right| 0\right\rangle \\
& =\frac{1}{2} i \int \mathrm{d} \mu^{2} \int \frac{\mathrm{d}^{4} k}{(2 \pi)^{4}} \frac{3 \rho_{A B}^{(1)}\left(\mu^{2}\right)}{k^{2}\left(k^{2}-\mu^{2}\right)} \\
& =-\frac{3}{32 \pi^{2}} \int \mathrm{d} \mu^{2} \ln \left(\frac{\Lambda^{2}}{\mu^{2}}\right) \rho_{A B}^{(1)}\left(\mu^{2}\right),
\end{aligned}
$$

where an ultraviolet cutoff $\Lambda$ has been introduced to regulate the momentum integral. A subtraction must be performed to define the vacuum expectation value of the time-ordered product of two currents. This subtraction can be construed as a redefinition of the zero of the effective potential $V(g)$ given by (3.6). After we subtract the (infinite) $\mathrm{G}_{\mathrm{f}}$-invariant part of $I^{A B}$, we can express $I^{A B}$ in terms of spectral integrals of the form

$$
\int \mathrm{d} \mu^{2} \ln \left(\frac{\Lambda^{2}}{\mu^{2}}\right) \rho\left(\mu^{2}\right)
$$

where the spectral function $\rho$, according to the reasoning of sect. 4 , obeys either the SFSR's

$$
\int \frac{\mathrm{d} s}{s} \rho(s)=F^{2}, \quad \int \mathrm{d} s \rho(s)=0
$$

or the SFSR's

$$
\int \frac{\mathrm{d} s}{s} \rho(s)=0, \quad \int \mathrm{d} s \rho(s)=0
$$

The second SFSR ensures that the coefficient of the logarithmic ultraviolet divergence in (5.2) vanishes.

In general, disregarding an irrelevant $G_{f}$-singlet part, we can express $V(g)$ in terms of $F$ 's, group-theoretic factors, and quantities of the form

$$
\Delta^{2}=\frac{1}{F^{2}} \int \mathrm{d} s \ln \left(\frac{s_{0}}{s}\right) \rho(s)
$$

where $\rho$ satisfies either (5.3) or (5.4). $\Delta^{2}$ is finite and independent of $s_{0}$.

In special cases to be considered later in this section, it is essential to determine the sign of $\Delta^{2}$ in order to distinguish minima of $V(g)$ from maxima. Unfortunately, 
(5.3) does not unambiguously fix the sign of $\Delta^{2}$. Both broken and unbroken currents couple to many vector resonances, so $\rho$ oscillates, taking both positive and negative values. However, we can determine the sign if it is a reasonable approximation to saturate the integral (5.5) with two low-lying narrow resonances. This approximation is known to be quite good in QCD, where it leads to successful predictions for the $A_{1}$ mass [15] and the electromagnetic mass difference of the pion [24]. We assume that spectral integrals are always rapidly convergent, so that saturating with two resonances makes sense.

If we take

$$
\rho(s)=g_{1}^{2} \delta\left(s-M_{1}^{2}\right)-g_{2}^{2} \delta\left(s-M_{2}^{2}\right),
$$

then eq. (5.3) implies that $g_{1}^{2}=g_{2}^{2}$ and that

$$
\frac{M_{1}^{2}}{M_{2}^{2}}=1-\frac{F^{2} M_{1}^{2}}{g_{1}^{2}} ;
$$

therefore, eq. (5.5) becomes

$$
\Delta^{2}=\frac{g_{1}^{2}}{F^{2}} \ln \left(\frac{M_{2}^{2}}{M_{1}^{2}}\right)>0 .
$$

Eq. (5.7) suggests that $F^{2} M_{1}^{2} / g_{1}^{2} \sim \frac{1}{2}$, and this relation can actually be "derived" by comparing soft Goldstone boson theorems with the results of a vector dominance approximation [25]. Therefore, when we need a numerical value for $\Delta^{2}$, we will use

$$
\Delta^{2}=2(\ln 2) M_{1}^{2}
$$

$M_{1}$ is the mass of the lightest vector resonance which couples to the currents associated with $\rho$.

If $\rho$ satisfies (5.4), then in the two-resonance approximation (5.6), we find $\rho=0$ and $\Delta^{2}=0$.

In the rest of this section, we will consider several special cases in which we can find explicit expressions for $V(g)$, and can make simple observations about the properties of the minimum.

\section{1. $\mathrm{G}_{\mathrm{f}}=\mathrm{SU}(N)_{\mathrm{L}} \times \mathrm{SU}(N)_{\mathrm{R}}, \mathrm{S}_{\mathrm{f}}=\mathrm{SU}(N)_{\mathrm{V}}$}

We first consider the case in which $\mathrm{G}_{\mathrm{f}}=\mathrm{SU}(N)_{\mathrm{L}} \times \mathrm{SU}(N)_{\mathrm{R}}$ chiral symmetry breaks down to $\mathrm{S}_{\mathrm{f}}=\mathrm{SU}(N)_{\mathrm{V}}$ isospin. This case is especially simple in that there is only one spectral integral on which the effective potential depends. We will see that $V(g)$ is proportional to a group-theoretic expression which has a simple interpretation. 
It is convenient to introduce notation in which all fermions transform as the same (complex) representation of $\mathrm{G}_{\mathrm{H}}$, so we have $N$ left-handed fermions and $N$ right-handed fermions. The $G_{f}$ currents are

$$
\begin{aligned}
& J_{\mathrm{L}_{\mu}}^{A}=\bar{\psi}_{\mathrm{L}} \gamma_{\mu} \lambda^{A} \psi_{\mathrm{L}}, \\
& J_{\mathrm{R}_{\mu}}^{A}=\bar{\psi}_{\mathrm{R}} \gamma_{\mu} \lambda^{A} \psi_{\mathrm{R}},
\end{aligned}
$$

where the $\lambda^{A}$ 's are hermitian traceless $N \times N$ matrices with flavor indices, normalized so that

$$
\operatorname{Tr} \lambda^{A} \lambda^{B}=\frac{1}{2}
$$

Both the left-handed and right-handed currents transform as the adjoint representation of $S_{f}$. The $G_{W}$ currents are

$$
\begin{aligned}
& J_{L_{\mu}}^{\alpha}=\bar{\psi}_{\mathrm{L}} \gamma_{\mu} \theta_{\mathrm{L}}^{\alpha} \psi_{\mathrm{L}}, \\
& J_{\mathrm{R}_{\mu}}^{\alpha}=\bar{\psi}_{\mathrm{R}} \gamma_{\mu} \theta_{\mathrm{R}}^{\alpha} \psi_{\mathrm{R}}
\end{aligned}
$$

under $G_{f}$ transformations they become

$$
\begin{aligned}
& J_{\mathrm{L} \mu}^{\alpha \alpha}=\bar{\psi}_{\mathrm{L}} \gamma_{\mu} U_{\mathrm{L}}^{\dagger} \theta_{\mathrm{L}}^{\alpha} U_{\mathrm{L}} \psi_{\mathrm{L}}, \\
& J_{\mathrm{R} \mu}^{\prime \alpha}=\bar{\psi}_{\mathrm{R}} \gamma_{\mu} U_{\mathrm{R}}^{\dagger} \theta_{\mathrm{R}}^{\alpha} U_{\mathrm{R}} \psi_{\mathrm{R}},
\end{aligned}
$$

where $U_{\mathrm{L}, \mathrm{R}}$ are $\mathrm{SU}(N)$ matrices.

Now $S_{\mathrm{f}}$ invariance can be exploited to find an explicit form for the effective potential. The effective potential is given by

$$
V(U)=-\frac{1}{2} i \int \mathrm{d}^{4} x \Delta^{\mu \nu}(x)\left(e_{\alpha}\right)^{2}\left\langle 0\left|\mathrm{~T}\left[J_{\mu}^{\prime \alpha}(x) J_{\nu}^{\prime \alpha}(0)\right]\right| 0\right\rangle
$$

with $J_{\mu}^{\prime \alpha}=J_{\mu \mathrm{L}}^{\prime \alpha}+J_{\mu \mathrm{R}}^{\prime \alpha}$. To calculate it, we extract the $\mathrm{S}_{\mathrm{f}}$ invariants from the timeordered product of $G_{W}$ currents. It is clear that $S_{f}$ invariance implies that

$$
\begin{aligned}
& \left\langle 0\left|J_{\mathrm{L}}^{\prime \alpha} J_{\mathrm{L}}^{\prime \alpha}\right| 0\right\rangle \propto \operatorname{Tr}\left(U_{\mathrm{L}}^{\dagger} \theta_{\mathrm{L}}^{\alpha} U_{\mathrm{L}} U_{\mathrm{L}}^{\dagger} \theta_{\mathrm{L}}^{\alpha} U_{\mathrm{L}}\right)=\operatorname{Tr} \theta_{\mathrm{L}}^{\alpha} \theta_{\mathrm{L}}^{\alpha}, \\
& \left\langle 0\left|J_{\mathrm{R}}^{\prime \alpha} J_{\mathrm{R}}^{\prime \alpha}\right| 0\right\rangle \propto \operatorname{Tr}\left(U_{\mathrm{R}}^{\dagger} \theta_{\mathrm{R}}^{\alpha} U_{\mathrm{R}} U_{\mathrm{R}}^{\dagger} \theta_{\mathrm{R}}^{\alpha} U_{\mathrm{R}}\right)=\operatorname{Tr} \theta_{\mathrm{R}}^{\alpha} \theta_{\mathrm{R}}^{\alpha}, \\
& \left\langle 0\left|J_{\mathrm{L}}^{\prime \alpha} J_{\mathrm{R}}^{\prime \alpha}\right| 0\right\rangle \propto \operatorname{Tr}\left(U_{\mathrm{L}}^{\dagger} \theta_{\mathrm{L}}^{\alpha} U_{\mathrm{L}} U_{\mathrm{R}}^{\dagger} \theta_{\mathrm{R}}^{\alpha} U_{\mathrm{R}}\right)=\operatorname{Tr}\left(\theta_{\mathrm{L}}^{\alpha} U \theta_{\mathrm{R}}^{\alpha} U^{\dagger}\right),
\end{aligned}
$$

where $U=U_{\mathrm{L}} U_{\mathrm{R}}^{\dagger}$ is an $\mathrm{SU}(N)$ matrix. The product of two left-handed or two 
right-handed currents contributes only an (infinite) $\mathrm{G}_{\mathrm{f}}$-invariant constant to $V(U)$. Subtracting this constant, we are left with

$$
\begin{aligned}
V(U) & =4\left(e_{\alpha}\right)^{2} \operatorname{Tr}\left(\theta_{\mathrm{L}}^{\alpha} U \theta_{\mathrm{R}}^{\alpha} U^{\dagger}\right)\left(-\frac{1}{2} i\right) \int \mathrm{d}^{4} x \Delta^{\mu \nu}(x) \underset{\text { (not summed over } B)}{\left\langle 0\left|\mathrm{~T}\left[J_{\mu \mathrm{L}}^{B}(x) J_{\nu}^{B}(0)\right]\right| 0\right\rangle} \\
& =-\frac{3}{32 \pi^{2}}\left(e_{\alpha}\right)^{2} \operatorname{Tr}\left(\theta_{\mathrm{L}}^{\alpha} U \theta_{\mathrm{R}}^{\alpha} U^{\dagger}\right) \int \mathrm{d} s \ln \left(\frac{s_{0}}{s}\right)\left[\rho_{\mathrm{V}}(s)-\rho_{\mathrm{A}}(s)\right]
\end{aligned}
$$

To obtain the second equality we have used eq. (5.1). The current $A_{\mu}^{B}=J_{L_{\mu}}^{B}-J_{R_{\mu}}^{B}$ couples to the Goldstone bosons with strength $F$, and the combination $\rho_{\mathrm{V}}-\rho_{\mathrm{A}}$ satisfies eq. (5.3). In terms of the positive quantity $\Delta^{2}$ defined by eq. (5.5), we have

$$
V(U)=-\frac{3}{32 \pi^{2}}\left(F^{2} \Delta^{2}\right)\left(e_{\alpha}\right)^{2} \operatorname{Tr} \theta_{\mathrm{L}}^{\alpha} U \theta_{\mathrm{R}}^{\alpha} U^{\dagger} .
$$

One can interpret eq. (5.17) by saying that the alignment of the subgroups $S_{f}$ and $G_{W}$ is determined by the condition that the breakdown of $G_{f}$ to $S_{f}$ occurs in the most attractive channel of the $G_{W}$ interactions. To see this, it is best to return to the notation in which all fermions are left-handed. The embedding of $S_{f}$ in $G_{f}$ can be characterized by a fermion condensate defined by

$$
\sigma \Sigma_{r s}=\left\langle 0\left|\varepsilon_{\alpha \beta} \lambda^{i j} \psi_{r i}^{\alpha} \psi_{s j}^{\beta}\right| 0\right\rangle,
$$

where $\lambda^{i j}$ is a $\mathrm{G}_{\mathrm{H}^{-}}$-invariant tensor, and the Lorentz indices are contracted by $\varepsilon_{\alpha \beta}$ to construct a Lorentz singlet; $\sigma$ is an overall scale factor. $S_{f}$ is the subgroup of $G_{f}$ which leaves $\Sigma$ invariant. If $\mathrm{G}_{\mathrm{f}}=\mathrm{SU}(N) \times \mathrm{SU}(N)$ and $\mathrm{S}_{\mathrm{f}}=\mathrm{SU}(N), \Sigma$ is a $2 N \times 2 N$ matrix of the form

$$
\Sigma=\left(\begin{array}{cc}
0 & U \\
U^{\mathbf{T}} & 0
\end{array}\right)
$$

and the $\mathbf{G}_{\mathbf{W}}$ generators can be written

$$
\theta^{\alpha}=\left(\begin{array}{cc}
\theta_{\mathrm{L}}^{\alpha} & 0 \\
0 & -\theta_{\mathrm{R}}^{\alpha *}
\end{array}\right)
$$

Expressed in terms of $\Sigma$ and $\theta^{\alpha}$, eq. (5.17) becomes

$$
V(\Sigma)=\frac{3}{64 \pi^{2}} F^{2} \Delta^{2}\left(e_{\alpha}\right)^{2} \Sigma_{r s}^{*} \theta_{r r^{\prime}}^{\alpha} \theta_{s s^{\prime}}^{\alpha} \Sigma_{r^{\prime} s^{\prime}}
$$

Because $F^{2} \Delta^{2}$ is positive, the alignment of the subgroups $\mathrm{G}_{\mathrm{w}}$ and $\mathrm{S}_{\mathrm{f}}$ is determined by the requirement that the value of the $\mathrm{G}_{\mathrm{w}}$ Casimir operator $\left(e_{\alpha}\right)^{2} \theta^{\alpha} \theta^{\alpha}$ acting on 


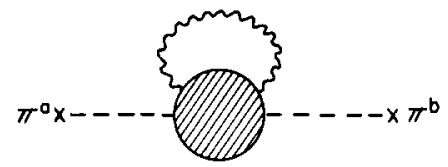

Fig. 4. Lowest-order $\mathbf{G}_{\mathrm{W}}$ contribution to $\mathrm{PGB}$ masses.

the condensate $\Sigma$ is as small as possible. In particular, if it is possible for the condensate to be oriented so that it is $G_{w}$ invariant, this orientation is always the minimum; the $\mathrm{G}_{\mathrm{w}}$ gauge group does not break unless it must.

By applying eq. (2.8) we find that the PGB mass matrix is (fig. 4)

$$
m_{A B}^{2}=-\frac{3}{8 \pi^{2}} \Delta^{2}\left(e_{\alpha}\right)^{2} \operatorname{Tr}\left[U^{\dagger} \theta_{\mathrm{L}}^{\alpha} U, \lambda^{A}\right]\left[\theta_{\mathrm{R}}^{\alpha}, \lambda^{B}\right] .
$$

Of course, the eigenvalues of this matrix are non-negative when $U$ is chosen to minimize $V(U)$.

\subsection{MAXIMAL ISOSPIN}

This case is a generalization of that of subsect. 5.1. We say that $S_{f}$ is maximal if $S_{f}$ is simple, all broken $G_{f}$ currents are in a single irreducible representation of $S_{f}$, and the commutator of two broken currents is an unbroken current. If $S_{f}$ is maximal, there is only one pair of SFSR's, and only one spectral integral on which the effective potential can depend. $V(g)$ can be expressed as a purely group-theoretic factor times a constant of known sign, as in eq. (5.17).

Aside from the breaking of $\mathrm{SU}(N) \times \mathrm{SU}(N)$ down to $\mathrm{SU}(N)$, there are two other notable examples of maximal $S_{f}$, both of which can occur only if the fermions are in a real representation of $G_{H}[6,26]$. Suppose there are $N$ flavors of fermions in a representation $R$ of $G_{H}$ such that the symmetric product of $R$ with itself contains the singlet. Then the tensor $\lambda^{i j}$ in eq. (5.18) is symmetric, and Fermi statistics requires $\Sigma_{r s}$ to be symmetric. (The fermions anticommute.) The maximal subgroup of $\mathrm{G}_{\mathrm{f}}=\mathrm{SU}(N)$ which leaves a symmetric tensor invariant is $\mathrm{S}_{\mathrm{f}}=\mathrm{O}(N)$. Under $\mathrm{O}(N)$, the adjoint representation of $\operatorname{SU}(N)$ splits up into two irreducible representations, an antisymmetric tensor (unbroken) and a traceless symmetric tensor (broken),

$$
N^{2}-1 \rightarrow \frac{1}{2} N(N-1)+\left[\frac{1}{2} N(N+1)-1\right]
$$

and the commutator of two broken currents is an unbroken current. [The case $N=4$ is exceptional; the broken currents are in the reducible representation $(1,3)+(3,1)$ of $\mathrm{O}(4)=\mathrm{SU}(2) \times \mathrm{SU}(2)$.]

If the fermions are in a representation $R$ of $G_{H}$ such that the antisymmetric product of $\mathbf{R}$ with itself contains the singlet, then $\Sigma_{r s}$ is antisymmetric. The 
maximal subgroup of $\mathrm{G}_{\mathrm{f}}=\mathrm{SU}(2 N)$ which leaves an antisymmetric tensor invariant is $\operatorname{Sp}(2 N)$. Under $\operatorname{Sp}(2 N)$, the adjoint representation of $\operatorname{SU}(2 N)$ splits up into two irreducible representations, a symmetric tensor (unbroken) and a "traceless" antisymmetric tensor (broken),

$$
4 N^{2}-1 \rightarrow N(2 N+1)+[N(2 N-1)-1],
$$

and the commutator of two broken currents is an unbroken current.

Assuming that $S_{\mathrm{f}}$ is maximal, we can apply eqs. (2.6)-(2.8) and (3.1) to derive the conditions satisfied at a local minimum of the effective potential. We first define an orthonormal basis for the $G_{f}$ generators such that

$$
\operatorname{Tr} X^{A} X^{B}=\operatorname{Tr} T^{A} T^{B}=\delta^{A B}, \quad \operatorname{Tr} X^{A} T^{B}=0,
$$

where $T^{A}$ 's are $\mathrm{S}_{\mathrm{f}}$ generators and the $X^{A}$ 's are broken generators. The broken currents $J_{X \mu}^{A}=\bar{\psi} \gamma_{\mu} X^{A} \psi$ couple to Goldstone bosons with strength $F$; that is

$$
\left\langle 0\left|J_{X_{\mu}}^{A}\right| \pi^{B}\right\rangle=i k_{\mu} F \delta^{A B}
$$

Given any embedding of $G_{W}$ in $G_{f}$, we can decompose the $G_{W}$ generators $\theta^{\alpha}$ into broken and unbroken generators, so that

$$
J_{\mu}^{\alpha}=\bar{\psi} \gamma_{\mu} \theta^{\alpha} \psi=\bar{\psi} \gamma_{\mu} T^{\alpha} \psi+\bar{\psi} \gamma_{\mu} X^{\alpha} \psi
$$

where

$$
\begin{aligned}
& T^{\alpha}=\sum_{A} T^{A} \operatorname{Tr}\left(T^{A} \theta^{\alpha}\right), \\
& X^{\alpha}=\sum_{A} X^{A} \operatorname{Tr}\left(X^{A} \theta^{\alpha}\right) .
\end{aligned}
$$

$T^{\alpha}$ and $X^{\alpha}$ vary in a complicated way under $\mathrm{G}_{\mathrm{f}}$ transformations.

The effective potential is stationary when

$$
\begin{aligned}
0 & =\left\langle 0\left|\left[\mathcal{H}^{\prime}, Q^{A}\right]\right| 0\right\rangle \\
& =-\frac{1}{2} i \int \mathrm{d}^{4} x \Delta^{\mu \nu}(x)\left(e_{\alpha}\right)^{2}\left\langle 0\left|\left[\mathrm{~T}\left(J_{\mu}^{\alpha}(x) J_{\nu}^{\alpha}(0)\right), Q^{A}\right]\right| 0\right\rangle,
\end{aligned}
$$

where

$$
Q^{A}=\int \mathrm{d}^{3} x J_{X_{0}}^{A}
$$


In an obvious shorthand, the commutator in eq. (5.27) is

$$
\begin{aligned}
{\left[\left(X^{\alpha}+T^{\alpha}\right)\left(X^{\alpha}+T^{\alpha}\right), X^{A}\right] } & =\left\{X^{\alpha},\left[X^{\alpha}, X^{A}\right]\right\}+\left\{X^{\alpha},\left[T^{\alpha}, X^{A}\right]\right\} \\
& =+\left\{T^{\alpha},\left[X^{\alpha}, X^{A}\right]\right\}+\left\{T^{\alpha},\left[T^{\alpha}, X^{A}\right]\right\} .
\end{aligned}
$$

Because the commutator of two broken generators is unbroken, and, of course, the commutator of a broken generator and an unbroken generator is broken, the first and last terms in eq. (5.29) contain no $S_{f}$ invariant, and have no vacuum expectation value. Noting that

$$
\operatorname{Tr} X^{\alpha}\left[T^{\alpha}, X^{A}\right]=-\operatorname{Tr} T^{\alpha}\left[X^{\alpha}, X^{A}\right]
$$

and invoking (5.1), eq. (5.27) becomes

$$
0=-\frac{3\left(e_{\alpha}\right)^{2}}{16 \pi^{2}} \operatorname{Tr}\left(T^{\alpha}\left[X^{\alpha}, X^{A}\right]\right) \int \mathrm{d} s \ln \left(\frac{s_{0}}{s}\right)\left(\rho_{T}-\rho_{X}\right),
$$

where $\rho_{T}$ and $\rho_{X}$ are the spectral functions associated with the unbroken and broken currents. Hence, the potential is stationary when a purely group-theoretic relation,

$$
\left(e_{\alpha}\right)^{2} \operatorname{Tr} T^{\alpha}\left[X^{\alpha}, X^{A}\right]=0
$$

is satisfied.

Similarly, we can compute the double commutator of eq. (2.8), retain only $S_{f}$ invariants, and obtain an expression for the PGB mass matrix. We find

$$
m_{A B}^{2}=\frac{3}{16 \pi^{2}} \Delta^{2}\left(e_{\alpha}\right)^{2}\left(-\operatorname{Tr}\left[T^{\alpha}, X^{A}\right]\left[T^{\alpha}, X^{B}\right]+\operatorname{Tr}\left[X^{\alpha}, X^{A}\right]\left[X^{\alpha}, X^{B}\right]\right),
$$

where

$$
\Delta^{2}=\frac{1}{F^{2}} \int \mathrm{d} s \ln \left(\frac{s_{0}}{s}\right)\left(\rho_{T}-\rho_{X}\right)
$$

In eq. (5.33), $\mathrm{m}^{2}$ has been expressed as the difference of two positive matrices. The unbroken $G_{W}$ generators give a positive contribution to $m^{2}$ and the broken $G_{W}$ generators give a negative contribution. At a local minimum of the potential, $\mathrm{m}^{2}$ must itself be positive. Roughly speaking, the minimum occurs when as large a subgroup of $\mathrm{G}_{\mathrm{w}}$ as possible is unbroken.

Only eqs. (5.32) and (5.33) will be needed to minimize the potential in the examples considered in sect. 6 , but the precise sense in which $G_{W}$ resists being 
broken can be clarified if we write down an explicit expression for the potential. If $S_{\mathrm{f}}$ is maximal, then the effective potential is given by

$$
\begin{aligned}
V & =-\frac{1}{2} i \int \mathrm{d}^{4} x \Delta^{\mu \nu}(x)\left(e_{\alpha}\right)^{2}\left\langle 0\left|\mathrm{~T}\left[J_{\mu}^{\alpha}(x) J_{\nu}^{\alpha}(0)\right]\right| 0\right\rangle \\
& =-\frac{3}{32 \pi^{2}}\left(e_{\alpha}\right)^{2}\left[C_{T} \operatorname{Tr}\left(T^{\alpha} T^{\alpha}\right)+C_{X} \operatorname{Tr}\left(X^{\alpha} X^{\alpha}\right)\right]
\end{aligned}
$$

where

$$
C_{T, X}=\int \mathrm{d} s \ln \left(\Lambda^{2} / s\right) \rho_{T, X}(s)
$$

To derive eq. (5.35), we have extracted the $S_{f}$ invariants from the product of two $\mathrm{G}_{\mathrm{W}}$ currents, and have invoked eq. (5.1). $T^{\alpha}$ and $X^{\alpha}$ depend implicitly on $g \in \mathrm{G}_{\mathrm{f}}$. Because

$$
\cot ^{2} \theta_{W}=g^{2} / g^{\prime 2}=\frac{1}{3}\left[4\left(q_{1}+q_{2}\right)^{2}-1\right]
$$

is $G_{f}$ invariant, the potential $V$ can be written

$$
V=\frac{3}{32 \pi^{2}}\left(F^{2} \Delta^{2}\right)\left(e_{\alpha}\right)^{2} \operatorname{Tr} X^{\alpha} X^{\alpha}+\text { constant },
$$

where $F^{2} \Delta^{2}=C_{T}-C_{X}>0$.

This expression for $V$ is non-negative. If it is possible to orient $G_{w}$ so that $X^{\alpha}=0$, that orientation minimizes $V . \mathrm{G}_{\mathrm{W}}$ will not break unless it must.

Recalling eq. (2.12), we see that the $G_{W}$ vector boson mass matrix is

$$
\mu_{\alpha \beta}^{2}=e_{\alpha} e_{\beta} F^{2} \operatorname{Tr}\left(X^{\alpha} X^{\beta}\right)
$$

(not summed). Therefore, the potential is

$$
V=\frac{3}{32 \pi^{2}} \Delta^{2}\left(\operatorname{Tr} \mu^{2}\right)
$$

The alignment of $G_{W}$ and $S_{f}$ is chosen to minimize the trace of the weak vector boson mass matrix. This condition defines the precise sense in which $G_{w}$ resists being broken, if $S_{f}$ is maximal.

Incidentally, one should not conclude from eq. (5.33) that it is impossible for $\mathbf{G}_{\mathrm{W}}$ to be completely broken when $S_{f}$ is maximal. Eq. (5.33) only implies that $T^{\alpha} \neq 0$ at the minimum; if all $G_{W}$ currents couple to Goldstone bosons, the currents must also have unbroken components which give a positive contribution to $\mathrm{m}^{2}$. 


\subsection{GENERALIZATIONS}

In the general case, the potential depends on many independent spectral integrals, and more detailed dynamical information is needed to determine the minimum. However, there are several generalizations of the case of maximal isospin for which we can still make some useful observations.

One generalization is trivial. If $G_{\mathrm{f}}=\Pi_{i} G_{i}$ breaks down to $S_{\mathrm{f}}=\Pi_{i} S_{i}$, where each $S_{i}$ is a maximal isospin subgroup of $G_{i}$, then all the $S_{i}$ 's align with $G_{W}$ independently, and the PGB masses are still given by (5.33), but with a different $\Delta_{i}^{2}$ for each $\mathbf{G}_{i}$.

We also observe that, if $S_{f}$ is simple and it is possible for $S_{f}$ and $G_{W}$ to align so that $G_{W}$ is unbroken, this alignment minimizes the potential. By a suitable orthogonal transformation, we can always define the $G_{f}$ currents so that the only $S_{f}$ invariants contributing to the potential are traces as in $(5.35)^{\star}$. We may then decompose the broken $\mathrm{G}_{\mathrm{W}}$ generators into generators in the different irreducible representations of $S_{f}^{\star \star}$,

$$
X^{\alpha}=\sum_{\mathbf{D}} X_{\mathrm{D}}^{\alpha}
$$

and, if $S_{f}$ is simple, we find that the potential is given by

$$
V=\frac{3}{32 \pi^{2}}\left(e_{\alpha}\right)^{2}\left[\sum_{\mathrm{D}}\left(F_{\mathrm{D}}^{2} \Delta_{\mathrm{D}}^{2}\right) \operatorname{Tr}\left(X_{\mathrm{D}}^{\alpha} X_{\mathrm{D}}^{\alpha}\right)\right]
$$

where

$$
F_{\mathrm{D}}^{2} \Delta_{\mathrm{D}}^{2}=\int \mathrm{d} s \ln \left(\frac{s_{0}}{s}\right)\left(\rho_{T}-\rho_{\mathrm{D}}\right)
$$

$V$ is non-negative, and is minimized by $X^{\alpha}=0$.

If $S_{\mathrm{f}}$ is not simple, there are additional terms in $V$ of the form

$$
\frac{3}{32 \pi^{2}}\left(e_{\alpha}\right)^{2}\left(C_{T}-C_{T^{\prime}}\right) \operatorname{Tr}\left(T^{\prime \alpha} T^{\prime \alpha}\right)
$$

where $T^{\alpha}$ and $T^{\prime \alpha}$ are components of the unbroken $\mathrm{G}_{\mathrm{W}}$ generators in two different factors of $S_{\mathrm{f}}$, and $C_{T, T^{\prime}}$ are defined as in (5.36). However, these terms are absent if we assume that spectral integrals can be well-approximated by saturating with two resonances. $C_{T}-C_{T^{\prime}}$ is a spectral integral of the form (5.5) where $\rho=\rho_{T}-\rho_{T^{\prime}}$ obeys the SFSR's (5.4). This spectral integral vanishes in the two-resonance approximation.

* We assume that this transformation does not mix unbroken currents and broken currents.

$\star \star A$ non-real irreducible representation of $S_{\mathbf{f}}$ plus its complex conjugate are regarded as a single representation in this decomposition. 


\section{Examples}

We now apply the general results of sect. 5 to eight specific models, with various choices of the groups $G_{f}, S_{f}$, and $G_{W}$. All of these examples are of genuine interest, because the chosen flavor groups are likely to be subgroups of the flavor group in a realistic hypercolor theory. The weak gauge groups we consider are also realistic. In examples (a)-(f), $\mathbf{G}_{\mathbf{W}}=\mathbf{S U}(2) \times \mathrm{U}(1)$ is the standard electroweak group. In example $(\mathrm{g}), \mathrm{G}_{\mathrm{w}}$ is $\mathrm{SU}(3) \times \mathrm{SU}(2) \times \mathrm{U}(1)$, where $\mathrm{SU}(3)$ is the color group. In example $(h), G_{w}=S U(3) \times S U(3)$ is a "chiral color" group.

We compute the dynamically determined pattern of $G_{w}$ breakdown for each sample model. Some models [examples (a), (e), (f)] are found to be phenomenologically unacceptable, because electric charge is broken.

The examples also illustrate an interesting general phenomenon. If the weak gauge group $G_{w}$ is not simple, the gauge interactions associated with the different factors of $G_{w}$ may be attractive in different channels, corresponding to different relative orientations of $G_{w}$ and $S_{f}$. Then these interactions compete, and which one wins depends on the relative strength of the gauge couplings. Hence, there are critical values of weak mixing angles at which the pattern of $G_{W}$ breaking changes discontinuously. This phenomenon occurs in examples (a) and (f), for which critical mixing angles are calculated.

We compute the electroweak and color contributions to the PGB masses for some of the examples. One important feature of the PGB masses was previously emphasized by Eichten and Lane [7]. If a model has a spontaneously broken approximate $\mathrm{SU}(N)_{\mathrm{L}} \times \mathrm{SU}(N)_{\mathrm{R}}$ chiral symmetry, and $\mathrm{G}_{\mathrm{W}}$ commutes with one of the chiral factors, then there will be PGB's which remain massless to lowest order in the $G_{w}$ interaction. Such a model might contain charged [example (d)] or colored [example (h)] PGB's which are much lighter than we would naively expect.

None of our sample models includes a sideways interaction [6-8]. We should bear in mind that this interaction is also expected to contribute to PGB masses in a truly realistic model. Eichten and Lane have estimated the sideways contribution to be $\Delta m^{2} \simeq(10-30 \mathrm{GeV})^{2}$.

(a) $\mathrm{Sp}$ (4) ISOSPIN

Suppose that four flavors of fermions are in the real irreducible representation $R$ of the hypercolor group $G_{H}$, where the antisymmetric product of $R$ with itself contains the $G_{H}$ singlet ${ }^{\star}$. The flavor group is $G_{f}=S U(4)$. The fermion condensate $\Sigma$ defined by eq. (5.18) is antisymmetric in flavor indices, and we assume that the maximal isospin group $\mathrm{S}_{\mathrm{f}}=\mathrm{Sp}(4)$ is left unbroken when hypercolor becomes strong. The $15 \mathrm{G}_{\mathrm{f}}$ currents transform as the 10 (unbroken) +5 (broken) representation of $S_{f}=S p(4)$.

\footnotetext{
* For example, $R$ could be the defining representation of $\mathrm{G}_{\mathrm{H}}=\mathrm{Sp}(2 n)$, or the $(2 n+1)$-index antisymmetric tensor representation of $\mathrm{G}_{\mathrm{H}}=\mathrm{SU}(4 n+2)$.
} 
Let the weak group be $G_{W}=S U(2) \times U(1)$, with the fermions in the $G_{W}$ representation

$$
\begin{array}{llll} 
& \left.\begin{array}{l}
N \\
E
\end{array}\right) & N^{\mathrm{c}} & E^{\mathrm{c}} \\
\frac{1}{2} Y= & -\frac{1}{2} & 0 & +1
\end{array}
$$

(All the fermions are left-handed.) These $U(1)_{\mathrm{W}}$ charge assignments are those we might expect for color-singlet hyperleptons. We will not worry about the fact that this model has a $\mathrm{G}_{\mathrm{W}}$ anomaly. The anomaly can be cancelled by additional fermions.

The effective potential is a function of the condensate $\Sigma$, which specifies the embedding of $S_{f}$ in $G_{f}$. Given $\Sigma$, we can express the $G_{w}$ generators as linear combinations of broken and unbroken $G_{f}$ generators. It is easy to verify using (5.32) that, if $S_{f}=S p(4)$, the effective potential for this model has only two extrema. These extrema are

$$
\left.\Sigma_{1}=\left(\begin{array}{rrrr}
N & E & N^{\mathrm{c}} & E^{\mathrm{c}} \\
0 & 0 & 1 & 0 \\
0 & 0 & 0 & 1 \\
-1 & 0 & 0 & 0 \\
0 & -1 & 0 & 0
\end{array}\right) \begin{array}{l}
N \\
N^{\mathrm{c}}
\end{array}, \quad \Sigma_{2}^{\mathrm{c}} \quad \begin{array}{rrrr}
0 & 1 & 0 & 0 \\
-1 & 0 & 0 & 0 \\
0 & 0 & 0 & 1 \\
0 & 0 & -1 & 0
\end{array}\right)
$$

$\Sigma_{1}$ breaks $G_{W}$ down to electric charge, but $\Sigma_{2}$ breaks $G_{W}$ down to $\operatorname{SU}(2)_{W}$. One of these is the minimum of the effective potential; the other is the maximum. But which is which?

To answer this question, we determine whether the PGB masses are positive or negative. If $\mathrm{Sp}(4)$ is defined by $\Sigma_{1}$, three of the five Goldstone bosons are eaten. Using (5.33), it is straightforward to calculate the mass of the uneaten PGB (and its $C P T$ conjugate). We find

$$
m_{1}^{2}=-\frac{9}{64 \pi^{2}}\left(g^{2}-g^{\prime 2}\right) \Delta^{2}
$$

where

$$
\Delta^{2}=\frac{1}{F^{2}} \int_{0}^{\infty} \mathrm{d} s \ln \left(\frac{s_{0}}{s}\right)\left(\rho_{10}-\rho_{5}\right)
$$

$g$ is the $\mathrm{SU}(2)_{\mathrm{W}}$ gauge coupling, and $g^{\prime}$ is the $\mathrm{U}(1)_{\mathrm{W}}$ gauge coupling. If the condensate is $\Sigma_{2}, \operatorname{SU}(2)_{\mathrm{w}}$ remains unbroken, and there are two uneaten PGB's. 
These have equal masses, given by

$$
m_{2}^{2}=-m_{1}^{2}
$$

We have discovered something interesting. The $\mathrm{SU}(2)_{\mathrm{W}}$ interaction is most attractive in the channel in which $\mathrm{SU}(2)_{\mathrm{W}}$ is unbroken, but the $\mathrm{U}(1)_{\mathrm{W}}$ interaction prefers to break $\mathrm{G}_{\mathrm{W}}$ to $\mathrm{U}(1)_{\mathrm{EM}}$. Thus, the two interactions compete, and which one wins depends on the relative strengths of the gauge couplings. At a critical value of the Weinberg mixing angle, the unbroken subgroup changes discontinuously from $\mathrm{SU}(2)$ to $\mathrm{U}(1)_{\mathrm{EM}}$; there is a first-order phase transition.

From eq. (6.3), we see that the critical Weinberg angle is

$$
\cot ^{2} \theta_{\mathrm{W}}=g^{2} / g^{2}=1 .
$$

We argued in sect. 5 that $\Delta^{2}$ is positive. Therefore, if $\cot ^{2} \theta_{W}<1$, then $m_{1}^{2}>0$, and $\mathrm{U}(1)_{\mathrm{EM}}$ is unbroken. If $\cot ^{2} \theta_{\mathrm{W}}>1$, then $m_{2}^{2}>0$, and $\mathrm{SU}(2)_{\mathrm{W}}$ is unbroken.

We can repeat the PGB mass calculation for different $U(1)_{W}$ charge assignments. If the charges are

$$
\begin{array}{ccc}
\left(\begin{array}{l}
N \\
E
\end{array}\right) & N^{\mathrm{c}} & E^{\mathrm{c}} \\
\frac{1}{2} Y=q & -\left(q+\frac{1}{2}\right) & -\left(q-\frac{1}{2}\right),
\end{array}
$$

then the critical Weinberg angle is

$$
\cot ^{2} \theta_{W}=g^{2} / g^{\prime 2}=\frac{1}{3}\left(16 q^{2}-1\right) .
$$

If $|q|<\frac{1}{4}$, the right-hand side of eq. (6.8) is negative, and no phase transition occurs at all; $\mathrm{SU}(2)_{\mathrm{W}}$ is always unbroken. The quark-like charge $q=\frac{1}{6}$ falls within this range.

There is a phase transition if $|q|>\frac{1}{4}$. In order to ensure that $\mathrm{G}_{\mathrm{w}}$ breaks to $\mathrm{U}(1)_{\mathrm{EM}}$ when the Weinberg angle has its observed value $\cot ^{2} \theta_{\mathrm{W}}=3.5$, we must have

$$
|q|>0.85
$$

The moral is that the breaking of an $\mathrm{SU}(4)$ chiral symmetry to $\mathrm{Sp}(4)$, with $\mathrm{G}_{\mathrm{W}} \subset \mathrm{SU}(4)$ embedded as in eq. (6.7), leads to the wrong kind of $\mathrm{G}_{\mathrm{w}}$ breaking unless the fermions have unconventional charges. This restriction should be kept in mind when model building is attempted.

(b) O(4) ISOSPIN

Again, we consider a model with four flavors of a real representation $R$ of $G_{H}$, with $\mathrm{G}_{\mathrm{W}}=\mathrm{SU}(2) \times \mathrm{U}(1) \subset \mathrm{SU}(4)$, and $\mathrm{G}_{\mathrm{W}}$ assignments as in eq. (6.1). But this 
time we suppose that the symmetric product of $\mathbf{R}$ with itself contains the singlet, and that the maximal isospin $O(4)$ is unbroken. Then we can show that the minimum of the effective potential is

$$
\Sigma=\left(\begin{array}{llll}
0 & 0 & 1 & 0 \\
0 & 0 & 0 & 1 \\
1 & 0 & 0 & 0 \\
0 & 1 & 0 & 0
\end{array}\right)
$$

In this case, it is impossible for $\mathrm{SU}(2)_{\mathrm{w}}$ to escape breaking, and there is no phase transition.

Under $\mathrm{O}(4)=\mathrm{SU}(2) \times \mathrm{SU}(2)$, the 15 of $\mathrm{SU}(4)$ transforms as $(1,3)+(3,1)+(3,3)$; there are nine Goldstone bosons. Because the currents are in three irreducible representations of $\mathrm{O}(4)$, this model has two independent pairs of SFSR's, and two spectral integrals on which the effective potential depends.

The unbroken currents are

$$
\begin{aligned}
& J_{1}^{+}=\sqrt{\frac{1}{2}}\left(\bar{E} N-\bar{N}^{\mathrm{c}} E^{\mathrm{c}}\right), \quad J_{2}^{+}=\sqrt{\frac{1}{2}}\left(\bar{E} N^{\mathrm{c}}-\bar{N} E^{\mathrm{c}}\right), \\
& J_{1}^{-}=\sqrt{\frac{1}{2}}\left(\bar{N} E-\bar{E}^{\mathfrak{c}} N^{\mathfrak{c}}\right), \quad J_{2}^{-}=\sqrt{\frac{1}{2}}\left(-\bar{N}^{\mathfrak{c}} E+\bar{E}^{\mathrm{c}} N\right), \\
& J_{1}^{3}=\frac{1}{2}\left(\bar{N} N-\bar{E} E-\bar{N}^{\mathrm{c}} N^{\mathrm{c}}+\bar{E}^{\mathrm{c}} E^{\mathrm{c}}\right), \quad J_{2}^{3}=\frac{1}{2}\left(\bar{N} N+\bar{E} E-\bar{N}^{\mathrm{c}} N^{\mathrm{c}}-\bar{E}^{\mathrm{c}} E^{\mathrm{c}}\right),
\end{aligned}
$$

and the broken currents are

$$
\begin{array}{rlrl}
K_{0}^{+} & =\bar{N} N^{\mathrm{c}}, & K_{1}^{+} & =\sqrt{\frac{1}{2}}\left(\bar{N} E^{\mathrm{c}}+\bar{E} N^{\mathrm{c}}\right), \\
K_{0}^{-} & =\bar{N}^{\mathrm{c}} N, & K_{2}^{+}=\bar{E} E^{\mathrm{c}}, \\
L^{+} & =\sqrt{\frac{1}{2}}\left(\bar{E} N+\bar{N}^{\mathrm{c}} E^{\mathrm{c}}\right), & \left.\bar{E}^{\mathrm{c}} N+\bar{N}^{\mathrm{c}} E\right), & K_{2}^{-}=\bar{E}^{\mathrm{c}} E, \\
L^{0} & =\frac{1}{2}\left(\bar{N} N-\overline{\frac{1}{2}}\left(\bar{N} E+\bar{E}^{\mathrm{c}} N^{\mathrm{c}}\right),\right.
\end{array}
$$

Here, and throughout this section, we exhibit the flavor structure of the currents, but suppress their Lorentz structure. We have displayed the currents of definite charge, rather than the hermitian currents. The corresponding SU(4) generators are normalized so that $\operatorname{Tr} \lambda^{A} \lambda^{B^{+}}=\delta^{A B}$. Each broken current couples to a Goldstone boson with strength $F$. 
The $G_{w}$ currents can be expressed as linear combinations of broken currents and unbroken currents; we have

$$
\begin{aligned}
J_{\mathrm{w}}^{+} & =\sqrt{\frac{1}{2}} \bar{E} N=\frac{1}{2}\left(L^{+}+J_{1}^{+}\right), \\
J_{\mathrm{w}}^{-} & =\sqrt{\frac{1}{2}} \bar{N} E=\frac{1}{2}\left(L^{-}+J_{1}^{-}\right), \\
J_{\mathrm{W}}^{3} & =\frac{1}{2}(\bar{N} N-\bar{E} E)=\frac{1}{2}\left(L^{0}+J_{1}^{3}\right), \\
J_{\mathrm{W}}^{Y / 2} & =-\frac{1}{2}(\bar{N} N+\bar{E} E)+\bar{E}^{\mathrm{c}} E^{\mathrm{c}}=-\frac{1}{2} L^{0}+\frac{1}{2} J_{1}^{3}-J_{2}^{3} .
\end{aligned}
$$

It is evident that the electromagnetic current $J_{\mathrm{W}}^{3}+J_{\mathrm{W}}^{Y / 2}$ is unbroken. Applying eq. (2.12), we see that the weak boson mass matrix is

which has the eigenvalues

$$
\mu^{2}=\left(\begin{array}{cccc}
g^{2} & 0 & 0 & 0 \\
0 & g^{2} & 0 & 0 \\
0 & 0 & g^{2} & g g^{\prime} \\
0 & 0 & g g^{\prime} & g^{\prime 2}
\end{array}\right) \frac{1}{4} F^{2}
$$

$$
\mu_{\mathrm{W}}^{2}=\frac{1}{4} g^{2} F^{2}, \quad \mu_{Z}^{2}=\frac{1}{4}\left(g^{2}+g^{\prime 2}\right) F^{2}, \quad \mu_{\gamma}^{2}=0 .
$$

The relation $\mu_{\mathrm{W}}^{2} / \mu_{\mathrm{Z}}^{2}=\cos ^{2} \theta_{\mathrm{W}}$ is enforced by the $\mathrm{O}(4)$ isospin.

The Goldstone bosons which are not eaten couple to the currents $K_{0,1,2}^{ \pm}$. The three PGB masses can be expressed in terms of the quantities

$$
\begin{aligned}
& \Delta_{1}^{2}=\frac{1}{F^{2}} \int \mathrm{d} s \ln \left(\frac{s_{0}}{s}\right)\left[\rho_{3(1)}-\rho_{9}\right], \\
& \Delta_{2}^{2}=\frac{1}{F^{2}} \int \mathrm{d} s \ln \left(\frac{s_{0}}{s}\right)\left[\rho_{3(2)}-\rho_{9}\right],
\end{aligned}
$$

where $\rho_{3(1)}$ and $\rho_{3(2)}$ are the spectral functions associated with $J_{1}$ and $J_{2}$. From (2.8) we find that the masses of the PGB's coupling to the currents $K_{0,1,2}$ are

$$
\begin{aligned}
& m_{0}^{2}=\frac{3}{64 \pi^{2}}\left[g^{2}\left(\frac{3}{2} \Delta_{1}^{2}-\frac{1}{2} \Delta_{2}^{2}\right)+g^{\prime 2}\left(2 \Delta_{2}^{2}-\Delta_{1}^{2}\right)\right], \\
& m_{1}^{2}=\frac{3}{64 \pi^{2}}\left[g^{2}\left(2 \Delta_{1}^{2}-\Delta_{2}^{2}\right)+3 g^{\prime 2} \Delta_{2}^{2}\right], \\
& m_{2}^{2}=\frac{3}{64 \pi^{2}}\left[g^{2}\left(2 \Delta_{1}^{2}-\Delta_{2}^{2}\right)+g^{\prime 2}\left(6 \Delta_{2}^{2}+3 \Delta_{1}^{2}\right)\right] .
\end{aligned}
$$


To obtain numerical values for the masses, we use the estimate in eq. (5.9),

$$
\Delta_{1}^{2}=\Delta_{2}^{2}=(2 \ln 2) M^{2},
$$

where $M$ is the mass of the lightest vector resonance which couples to the unbroken $\mathrm{O}(4)$ currents. Roughly, we expect $M \simeq\left(F / f_{\pi}\right) m_{\rho}$, and $\mu_{\mathrm{W}}^{2}=\frac{1}{4} g^{2} F^{2}$ implies $F \simeq 250$ $\mathrm{GeV}$; therefore, we have $M \simeq 2.0 \mathrm{TeV}$ and

$$
\begin{aligned}
& m_{0}^{2}=\frac{3(\ln 2) \alpha}{2 \pi \sin ^{2} 2 \theta_{\mathrm{W}}} M^{2} \simeq(120 \mathrm{GeV})^{2}, \\
& m_{1}^{2}=m_{0}^{2}\left(1+2 \sin ^{2} \theta_{\mathrm{w}}\right) \simeq(140 \mathrm{GeV})^{2}, \\
& m_{2}^{2}=m_{0}^{2}\left(1+8 \sin ^{2} \theta_{\mathrm{w}}\right) \simeq(200 \mathrm{GeV})^{2},
\end{aligned}
$$

taking $\sin ^{2} \theta_{\mathrm{W}} \simeq 0$.23. In a realistic model, more $\mathrm{G}_{\mathrm{H}}$ representations will contribute to $\mu_{\mathrm{W}}, \mu_{\mathrm{Z}}$, and $F$ will be smaller than $250 \mathrm{GeV}$. The PGB masses scale with $F$.

(c) O(3) ISOSPIN

Now we suppose that there are three real representations of $G_{H}$, with $G_{W}$ representation content given by

$$
\begin{array}{lll} 
& \left(\begin{array}{c}
N \\
E
\end{array}\right) & E^{\mathrm{c}} \\
\frac{1}{2} Y=\quad-\frac{1}{2} & 1 .
\end{array}
$$

This structure might arise if the $N^{\mathrm{c}}$, which is not protected by $\mathrm{G}_{\mathrm{w}}$ quantum numbers, gets a large Majorana mass before the $G_{H}$ interaction gets strong [27].

Suppose that the condensate is symmetric in flavor indices, and breaks $G_{f}=S U(3)$ down to $S_{f}=O(3)$. Then we can verify that the condensate which minimizes the potential is

$$
\Sigma=\left(\begin{array}{ccc}
N & E & E^{\mathrm{c}} \\
1 & 0 & 0 \\
0 & 0 & 1 \\
0 & 1 & 0
\end{array}\right)_{E^{\mathrm{c}}}^{N},
$$

which breaks $\mathrm{G}_{\mathrm{W}}$ to $\mathrm{U}(1)_{\mathrm{EM}}$.

The Goldstone bosons transform as a 5 under $O(3)$ isospin. Therefore, isospin does not enforce the natural $\mu_{\mathrm{w}} / \mu_{\mathrm{Z}}$ ratio. Instead we find

$$
\mu_{\mathrm{w}}^{2} / \mu_{\mathrm{Z}}^{2}=\frac{2}{3} \cos ^{2} \theta_{\mathrm{W}}
$$


If this type of flavor symmetry breaking is to be allowed in a realistic model, there must be many other Goldstone boson representations contributing to the $\mathrm{W}$ and $\mathrm{Z}$ masses which swamp the contribution of the $O(3)$ 5-plet.

(d) SU(2N) ISOSPIN

Next we consider a model with fermions in complex representations of $\mathbf{G}_{\mathbf{H}}$. Suppose we have $N$ left-handed $\mathrm{G}_{\mathrm{w}}$ doublets and $2 N$ right-handed singlets, with $\mathrm{U}(1)_{\mathrm{W}}$ charge assignments given by

$$
\begin{aligned}
& \left(\begin{array}{c}
U_{i} \\
D_{i}
\end{array}\right)_{\mathrm{L}} \quad U_{i \mathrm{R}} \quad D_{i \mathrm{R}} \\
& \frac{1}{2} Y=\quad q \quad q+\frac{1}{2} \quad q-\frac{1}{2} .
\end{aligned}
$$

The approximate chiral symmetry group is $\mathrm{G}_{\mathrm{f}}=\mathrm{SU}(2 N)_{\mathrm{L}} \times \mathrm{SU}(2 N)_{\mathrm{R}}$, which we assume breaks down to $\mathrm{SU}(2 N)_{\mathrm{v}}$.

If $N=1$, all three Goldstone bosons are eaten by the $\mathrm{W}^{ \pm}$and $\mathrm{Z}$, so there is no subgroup alignment problem to solve; all $G_{\mathrm{f}}$ rotations of the condensate are $G_{W}$ gauge transformations. If $N>1$, the global symmetry group respected by the $\mathrm{G}_{\mathrm{W}}$ interactions is $\mathrm{G}_{\mathrm{f}}^{\prime}=\mathrm{SU}(N) \times \mathrm{SU}(N) \times \mathrm{SU}(N)$ which breaks down to the exact isospin $\mathrm{S}_{\mathrm{f}}^{\prime}=\mathrm{SU}(N)$. Therefore, there are $2\left(N^{2}-1\right)$ (electrically neutral) exactly massless Goldstone bosons. In a realistic model, the sideways interaction is needed to provide masses for these Goldstone bosons [7].

The breakdown of the approximate $\mathrm{SU}(2 N) \times \mathrm{SU}(2 N)$ chiral symmetry produces $4 N^{2}-1$ Goldstone bosons, of which 3 are eaten. Hence there are $2\left(N^{2}-1\right)$ remaining charged PGB's which should receive mass from the $G_{w}$ interactions. However, it is easy to see that the lowest-order $G_{w}$ interactions give no contribution to these PGB masses [7]. As we observed in sect. 5, the lowest-order $G_{w}$ contribution to the effective potential has the form

$$
\begin{aligned}
V(g)= & -i\left(e_{\alpha}\right)^{2} \int \mathrm{d}^{4} x \Delta^{\mu \nu}(x)\left\langle 0\left|\mathrm{~T}\left[J_{L_{\mu}}^{\prime \alpha}(x) J_{R \nu}^{\prime \alpha}(0)\right]\right| 0\right\rangle \\
& + \text { constant } .
\end{aligned}
$$

Because the $\mathrm{SU}(2)_{\mathrm{W}}$ interactions are purely left-handed, they do not contribute to $V$. (We can say this another way. The Goldstone bosons couple to axial currents, but we can use right-handed currents as their interpolating currents. The $S U(2)_{w}$ currents commute with these interpolating currents, so the $\mathrm{SU}(2)_{\mathrm{w}}$ interaction cannot give mass to the Goldstone bosons.) The $\mathrm{U}(1)_{\mathrm{w}}$ contribution to $V$ also vanishes, because the left-handed $\mathrm{U}(1)_{\mathrm{W}}$ current is an $\mathrm{SU}(2 N)_{\mathrm{L}}$ singlet; this contribution is $\mathrm{SU}(2 N)_{\mathrm{L}}$ invariant as well as $\mathrm{SU}(2 N)_{\mathbf{V}}$ invariant, and is therefore invariant under all $G_{f}$ chiral transformations. 
a)

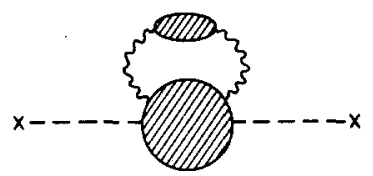

b)

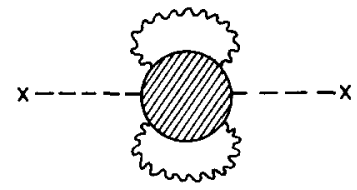

c)

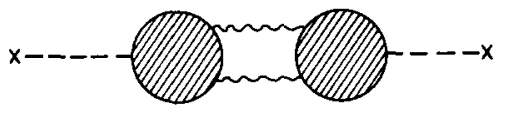

d)

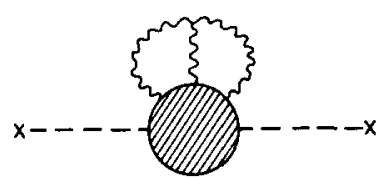

Fig. 5. Higher-order $G_{w}$ contributions to PGB mass.

Because the masslessness of the charged PGB's is not enforced by any exact symmetry, it will be removed by higher-order $\mathbf{G}_{\mathbf{w}}$ corrections. The higher-order corrections can be estimated by taking into account the mass splitting of the $\gamma$ and $Z$ which is generated in the next order (fig. $5 \mathrm{a}$ ). Then the minimum of $V$ is found to occur when all fermion generations line up to leave $\mathrm{U}(1)_{\mathrm{EM}}$ unbroken. To calculate the PGB masses we can use the currents

$$
\begin{aligned}
& J_{\mathrm{R} i j}^{+}=\sqrt{2} \bar{D}_{\mathrm{R} i} U_{\mathrm{R} j}, \\
& J_{\mathrm{R} i j}^{-}=\sqrt{2} \bar{U}_{\mathrm{R} j} D_{\mathrm{R} i},
\end{aligned}
$$

which couple to the Goldstone bosons with strength $F$. The currents coupling to $\gamma$ and $Z$ can be written

$$
\begin{array}{ll}
J_{\mathrm{R}}^{\gamma}=g^{\prime} J_{\mathrm{R}}^{3} \cos \theta+\text { singlet }, & J_{\mathrm{R}}^{\mathrm{Z}}=-g^{\prime} J_{\mathrm{R}}^{3} \sin \theta+\text { singlet }, \\
J_{\mathrm{L}}^{\gamma}=g J_{\mathrm{L}}^{3} \sin \theta+\operatorname{singlet}, & J_{\mathrm{L}}^{\mathrm{Z}}=g J_{\mathrm{L}}^{3} \cos \theta+\text { singlet },
\end{array}
$$

where $J^{3}=\Sigma_{i} \frac{1}{2}\left(\bar{U}_{i} U_{i}-\bar{D}_{i} D_{i}\right)$ and "singlet" represents $\mathrm{G}_{\mathrm{f}}$-invariant terms. Now we 
can use eq. (2.8) to calculate the masses of the charged PGB's. We find

$$
\begin{aligned}
m^{2} & =\frac{e^{2}}{F_{2}} i \int \mathrm{d}^{4} x\left(\Delta_{\mu \nu}^{\gamma}(x)-\Delta_{\mu \nu}^{\mathrm{Z}}(x)\right)\left\langle 0\left|\mathrm{~T}\left[V^{\mu}(x) V^{\nu}(0)-A^{\mu}(x) A^{\nu}(0)\right]\right| 0\right\rangle \\
& =\frac{3 e^{2}}{16 \pi^{2} F^{2}} \int_{0}^{\infty} \mathrm{d} s\left[\ln \left(\frac{s_{0}}{s}\right)-\frac{s}{s-\mu_{\mathrm{Z}}^{2}} \ln \left(\frac{\mu_{\mathrm{Z}}^{2}}{s}\right)\right]\left(\rho_{\mathrm{V}}-\rho_{\mathrm{A}}\right),
\end{aligned}
$$

where $\rho_{\mathrm{V}}$ and $\rho_{\mathrm{A}}$ are the vector and axial vector spectral functions.

We can use eq. (6.25) to calculate the electromagnetic mass difference of the ordinary pion. Then we saturate the spectral integral with the $\rho$ and $A_{1}$ resonances; the second term is suppressed by $m_{\rho}^{2} / \mu_{Z}^{2}$, and it is sensible to ignore it. Estimating the spectral integral as in (5.9) we have

$$
m_{+}^{2}-m_{0}^{2}=\frac{3 e^{2}}{16 \pi^{2} F^{2}} \int_{0}^{\infty} \mathrm{d} s \ln \left(\frac{s_{0}}{s}\right)\left(\rho_{\mathrm{V}}-\rho_{\mathrm{A}}\right) \simeq \frac{3 \ln 2}{2 \pi} \alpha m_{\rho}^{2} .
$$

This is the classic formula of Das et al. [24], which is known to be well satisfied.

In the case of interest to us here, we want to saturate the spectral integral with hypermeson resonances $M_{\mathrm{V}}, M_{\mathrm{A}} \gg \mu_{\mathrm{Z}}$. Therefore, we have

$$
\begin{aligned}
m^{2} & \simeq \frac{3 e^{2}}{16 \pi^{2} F^{2}} \int \mathrm{d} s \frac{\mu_{\mathrm{Z}}^{2}}{s} \ln \left(\frac{s}{\mu_{\mathrm{Z}}^{2}}\right)\left(\rho_{\mathrm{V}}-\rho_{\mathrm{A}}\right) \\
& \simeq \frac{3 \alpha}{4 \pi} \mu_{\mathrm{Z}}^{2} \ln \left(\frac{M_{\mathrm{V}}^{2}}{2 \mu_{\mathrm{Z}}^{2}}\right) \simeq(7 \mathrm{GeV})^{2}
\end{aligned}
$$

where we have used eq. (5.6) to obtain the second equality in eq. (6.27), and have taken $M_{\mathrm{V}} \simeq 1 \mathrm{TeV}$.

Unlike the PGB mass in example (b), this mass does not scale with $F$; the dependence on the hypercolor scale is only in the logarithm ${ }^{\star}$. Therefore eq. (6.27) should be a fairly reliable estimate of the electroweak contribution to the mass of these PGB's. As Eichten and Lane [7] have emphasized, this prediction is quite exciting, because relatively light charged PGB's could be produced in $\mathrm{e}^{+} \mathrm{e}^{-}$ colliding beam experiments very soon.

The $2\left(N^{2}-1\right)$ charged PGB's are still degenerate. This degeneracy will not be lifted in higher order. The positively charged PGB's, and their CPT conjugates, are in $\left(N^{2}-1\right)$ multiplets of the exact unbroken $\mathrm{SU}(N)$ isospin.

\footnotetext{
* The graphs in fig. $5 \mathrm{~b}-\mathrm{d}$ have been ignored in our derivation of (6.27). Eichten and Lane [7] conjectured, and Peskin [14] has explicitly verified, that the coefficient of the logarithm in (6.27) is correctly accounted for by fig. $5 \mathrm{a}$ alone, at least in the model considered here. The additional graphs only modify the argument of the logarithm.
} 
(e) TWO LEFT-HANDED DOUBLETS

In example (d), all $N$ left-handed $\mathrm{SU}(2)_{\mathrm{w}}$ doublets had the same value of the $\mathrm{U}(1)_{\mathrm{W}}$ charge $\frac{1}{2} Y$. But if there are left-handed doublets with different values of $\frac{1}{2} Y$, then the $U(1)_{W}$ interaction contributes to the effective potential in lowest order, and the pattern of $G_{W}$ breakdown depends on the $U(1)_{W}$ charge assignments.

Consider a model with four left-handed and four right-handed fermions in a complex representation of $G_{H}$, and with $G_{W}$ representation content given by

$$
\begin{aligned}
& \left(\begin{array}{c}
U_{1} \\
D_{1}
\end{array}\right)_{\mathrm{L}}\left(\begin{array}{c}
U_{2} \\
D_{2}
\end{array}\right)_{\mathrm{L}} \quad U_{\mathrm{IR}} \quad D_{\mathrm{IR}} \quad U_{2 \mathrm{R}} \quad D_{2 \mathrm{R}} \\
& \frac{1}{2} Y=\quad q_{1} \quad q_{2} \quad q_{1}+\frac{1}{2} \quad q_{1}-\frac{1}{2} \quad q_{2}+\frac{1}{2} \quad q_{1}-\frac{1}{2} .
\end{aligned}
$$

$\left(\mathrm{G}_{\mathrm{W}}\right.$ anomalies can be eliminated by choosing $q_{1}=-q_{2}$ or by adding more fermions.)

We assume that the $G_{f}=S U(4)_{L} \times S U(4)_{R}$ chiral symmetry breaks down to $S_{f}=S U(4)_{V}$. The embedding of $S_{f}$ in $G_{f}$ can be specified by a fermion condensate $\mathrm{W}$ defined by

$$
\left\langle 0\left|\bar{\psi}_{R s} \psi_{L r}\right| 0\right\rangle=\sigma W_{r s}
$$

$W$ is a unimodular unitary matrix. The dependence of the effective potential $V$ on $W$ is given by eq. (5.17). As in example (d), the $\mathrm{SU}(2)_{\mathrm{w}}$ interaction does not contribute to $V(W)$ in lowest order.

If $q_{1}>q_{2}$, the minimum of the effective potential occurs at one of the two stationary points

$$
\begin{aligned}
& \bar{U}_{1 \mathrm{R}} \quad \bar{D}_{1 \mathrm{R}} \quad \bar{U}_{2 \mathrm{R}} \quad \bar{D}_{2 \mathrm{R}} \\
& W_{1}=\left(\begin{array}{llll}
1 & 0 & 0 & 0 \\
0 & 1 & 0 & 0 \\
0 & 0 & 1 & 0 \\
0 & 0 & 0 & 1
\end{array}\right) \begin{array}{l}
U_{1 \mathrm{~L}} \\
D_{1 \mathrm{~L}} \\
U_{2 \mathrm{~L}} \\
D_{2 \mathrm{~L}}
\end{array}, \quad W_{2}=\left(\begin{array}{llll}
1 & 0 & 0 & 0 \\
0 & 0 & 1 & 0 \\
0 & 1 & 0 & 0 \\
0 & 0 & 0 & 1
\end{array}\right)
\end{aligned}
$$

$W_{1}$ leaves $\mathrm{U}(1)_{\mathrm{EM}}$ unbroken, but $W_{2}$ breaks $\mathrm{G}_{\mathrm{W}}$ completely. From (5.17), we find the value of the effective potential at these points to be

$$
\begin{aligned}
& V\left(W_{1}\right)=\frac{-3}{32 \pi^{2}} F^{2} \Delta^{2} g^{\prime 2}\left(2 q_{1}^{2}+2 q_{2}^{2}\right), \\
& V\left(W_{2}\right)=\frac{-3}{32 \pi^{2}} F^{2} \Delta^{2} g^{\prime 2}\left[\left(q_{1}+q_{2}\right)^{2}+q_{1}-q_{2}\right],
\end{aligned}
$$


and therefore we have

$$
V\left(W_{1}\right)-V\left(W_{2}\right)=\frac{-3}{32 \pi^{2}} F^{2} \Delta^{2} g^{\prime 2}\left[\left(q_{1}-q_{2}\right)\left(q_{1}-q_{2}-1\right)\right]
$$

Hence if

$$
0<\left|q_{1}-q_{2}\right|<1
$$

$W_{2}$ minimizes the effective potential, and $G_{w}$ is completely broken.

In this model, although there exists a relative orientation of $G_{w}$ and $S_{f}$ which leaves $\mathrm{U}(1)_{\mathrm{EM}}$ unbroken, $\mathrm{G}_{\mathrm{W}}$ is completely broken in the dynamically preferred vacuum if (6.33) is satisfied.

(f) RIGHT-HANDED DOUBLETS

If a representation of the sideways group $G_{S}$ [6-8] contains a complex representation $R$ of $G_{H} \subset G_{S}$, it may also contain the conjugate representation $\bar{R}$. Therefore, some hypercolor models contain both left-handed and right-handed $G_{W}$ doublets. Consider, then, a model with four left-handed and four right-handed fermions in a complex representation of $G_{H}$, and with the $G_{w}$ representation content given by

$$
\begin{aligned}
& \left(\begin{array}{c}
U_{1} \\
D_{1}
\end{array}\right)_{\mathrm{L}} \quad U_{1 \mathrm{R}} \quad D_{1 \mathrm{R}} \quad\left(\begin{array}{c}
U_{2} \\
D_{2}
\end{array}\right)_{\mathrm{R}} \quad U_{2 \mathrm{~L}} \quad D_{2 \mathrm{~L}} \\
& \frac{1}{2} Y=\quad q_{1} \quad q_{1}+\frac{1}{2} \quad q_{1}-\frac{1}{2} \quad-q_{2}-\left(q_{2}+\frac{1}{2}\right) \quad-\left(q_{2}-\frac{1}{2}\right) .
\end{aligned}
$$

This model is similar to example (a) in that the effective potential has two possible minima, at one of which $\mathrm{SU}(2)_{\mathrm{w}}$ is left unbroken. The $\mathrm{SU}(2)_{\mathrm{w}}$ interaction is most attractive in the $\mathrm{SU}(2)_{\mathrm{W}}$ singlet channel, while the $\mathrm{U}(1)_{\mathrm{W}}$ interaction is more attractive in the channel in which $\mathrm{U}(1)_{\mathrm{EM}}$ is unbroken. Therefore, the two interactions compete, and, as in example (a), there is a critical value of the Weinberg angle at which a first-order phase transition occurs.

We assume again that the $G_{f}=S U(4)_{L} \times S U(4)_{R}$ chiral symmetry breaks down to $\mathrm{S}_{\mathrm{f}}=\mathrm{SU}(4)_{\mathrm{V}}$. The effective potential $V(W)$ given by $(5.17)$ has stationary points at

$$
\begin{aligned}
& \bar{U}_{1 \mathrm{R}} \quad \bar{D}_{1 \mathrm{R}} \quad \bar{U}_{2 \mathrm{R}} \quad \bar{D}_{2 \mathrm{R}} \\
& W_{1}=\left(\begin{array}{llll|l}
1 & 0 & 0 & 0 \\
0 & 1 & 0 & 0 & U_{1 \mathrm{~L}} \\
0 & 0 & 1 & 0 & D_{1 \mathrm{~L}} \\
U_{2 \mathrm{~L}} \\
D_{2 \mathrm{~L}}
\end{array}, \quad W_{2}=\left(\begin{array}{llll}
0 & 0 & 1 & 0 \\
0 & 0 & 0 & 1 \\
0 & 1 & 0 & 0 \\
1 & 0 & 0 & 0
\end{array}\right) .\right.
\end{aligned}
$$

$W_{1}$ leaves $U(1)_{\mathrm{EM}}$ unbroken and $W_{2}$ leaves $\mathrm{SU}(2)_{\mathrm{W}}$ unbroken. From (5.17), we find that

$$
V\left(W_{1}\right)-V\left(W_{2}\right)=\frac{3}{64 \pi^{2}} F^{2} \Delta^{2}\left\{3 g^{2}-\left[4\left(q_{1}+q_{2}\right)^{2}-1\right] g^{\prime 2}\right\}
$$


Therefore, $W_{1}$ is the minimum of the potential, and $\mathrm{G}_{\mathrm{W}}$ breaks down to $\mathrm{U}(1)_{\mathrm{EM}}$ only if $\cot ^{2} \theta_{\mathrm{w}}$ is smaller than a critical value given by

$$
\cot ^{2} \theta_{W}=g^{2} / g^{\prime 2}=\frac{1}{3}\left[4\left(q_{1}+q_{2}\right)^{2}-1\right] .
$$

There is no phase transition if $\left|q_{1}+q_{2}\right|>\frac{1}{2}$; $\mathrm{SU}(2)_{\mathrm{W}}$ is always unbroken. For lepton-like charges $q_{1}=q_{2}=-\frac{1}{2}$, the critical Weinberg angle is $\cot ^{2} \theta_{\mathrm{W}}=1$. To ensure that $\mathrm{G}_{\mathrm{W}}$ breaks down to $\mathrm{U}(1)_{\mathrm{EM}}$ when $\sin ^{2} \theta=0.23$, we must have $\left|q_{1}+q_{2}\right|$ $>1.7$.

If we add more left-handed $G_{W}$ doublets and right-handed singlets to the model, they do not help to stabilize the vacuum in which $\mathrm{U}(1)_{\mathrm{EM}}$ is unbroken. They $d o$ break $S U(2)_{W}$, so if we have, for example, a model with two left-handed doublets and one right-handed doublet, plus singlets, $G_{W}$ is completely broken. This occurs even though there is a relative orientation of $G_{w}$ and $S_{f}$ which leaves $U(1)_{E M}$ unbroken.

Therefore, right-handed $G_{w}$ doublets are very dangerous. Unless they have unconventionally large electric charges, they refuse to allow $G_{w}$ to break in the observed way.

(g) WEAK COLOR

When hypercolor becomes strong at a renormalization scale near $1 \mathrm{TeV}$, the color coupling is $\alpha_{c} \simeq 0.1$, so $\mathrm{SU}(3)_{\mathrm{c}}$ can be treated as a chiral-symmetry-breaking perturbation too. The weakly gauged subgroup of $G_{f}$ is really $G_{w}=S U(3) \times S U(2)$ $\times U(1)$. As we saw that $S U(2)$ and $U(1)$ may compete with each other to determine the alignment of $G_{w}$ with $S_{f}$, so $S U(3)_{c}$ may compete with the electroweak group. If so, color will win because $\alpha_{\mathrm{c}}$ is large compared to $\alpha$.

For instance, suppose that the hyperfermions in example (a) come in three colors; $N$ and $E$ are 3's but $N^{\mathrm{c}}$ and $E^{\mathrm{c}}$ are $\overline{3}$ 's. The flavor symmetry is now $\mathrm{G}_{\mathrm{f}}=\mathrm{SU}(12)$; we assume it breaks down to $\mathrm{S}_{\mathrm{f}}=\mathrm{Sp}(12)$. As before, the electroweak interactions will prefer that $S U(2)_{W}$ be unbroken, but color will favor the colorsinglet channel, and insist that $\mathrm{SU}(2) \times \mathrm{U}(1)$ break down to $\mathrm{U}(1)_{\mathrm{EM}}$.

In any realistic model, there are many PGB's which carry color, and we wish to estimate their masses. Therefore, we consider a model with fermions in a complex representation of $G_{H}$, and $G_{W}=S U(3) \times S U(2) \times U(1)$ representation content given by ${ }^{\star}$

$$
\frac{1}{2} Y=\begin{array}{cccccc}
\left(\begin{array}{c}
U_{i} \\
D_{i}
\end{array}\right)_{\mathrm{L}} & U_{i \mathrm{R}} & D_{i \mathrm{R}} & \left(\begin{array}{c}
N \\
E
\end{array}\right)_{\mathrm{L}} & N_{\mathrm{R}} & E_{\mathrm{E}}, \\
\frac{1}{6} & \frac{2}{3} & -\frac{1}{3} & -\frac{1}{2} & 0 & -1
\end{array}
$$

where $U, D$ are color triplets and $N, E$ are color singlets. This model has an approximate $\mathrm{G}_{\mathrm{f}}=\mathrm{SU}(8)_{\mathrm{L}} \times \mathrm{SU}(8)_{\mathrm{R}}$ chiral symmetry which we assume breaks down to $\mathrm{S}_{\mathrm{f}}=\mathrm{SU}(8)_{\mathrm{v}}$.

\footnotetext{
* The PGB spectrum of this model has also been considered by Peskin [14] and Dimopoulos [26]. See also Farhi and Susskind [28].
} 
The subgroup of $S_{f}$ respected by the color interaction is $\mathrm{SU}(3)_{\mathrm{c}} \times \mathrm{SU}(2)$; the right-handed and left-handed fermions transform as the $(3,2)+(1,2)$ representation of this group. The $\mathrm{SU}(2)$ isospin is further broken to electric charge by the electroweak interaction. Under $\mathrm{SU}(3) \times \mathrm{SU}(2)$, the 63 Goldstone bosons transform as

$$
63=(8,3)+(8,1)+(1,3)+(1,3)+(1,1)+(3,3)+(\overline{3}, 3)+(3,1)+(\overline{3}, 1) .
$$

A combination of the $(1,3)$ 's is eaten by the $\mathrm{W}^{ \pm}$and $Z$. The $(1,1)$ and the neutral member of the remaining $(1,3)$ are exactly massless Goldstone bosons [7]. The charged members of the uneaten $(1,3)$ receive masses of order $7 \mathrm{GeV}$ from the second-order electroweak interactions, as in example (d).

The remaining Goldstone bosons are colored, and receive masses from the lowest-order color interaction. Using eq. (5.22), we find that the color-octet and color-triplet PGB masses are

$$
\begin{aligned}
& m_{8}^{2}=\frac{3 g_{c}^{2}}{16 \pi^{2}} c_{2}(8) \Delta^{2}, \\
& m_{3}^{2}=\frac{3 g_{c}^{2}}{16 \pi^{2}} c_{2}(3) \Delta^{2},
\end{aligned}
$$

where

$$
\Delta^{2}=\frac{1}{F^{2}} \int_{0}^{\infty} \mathrm{d} s \ln \left(\frac{s_{0}}{s}\right)\left(\rho_{\mathrm{V}}-\rho_{\mathrm{A}}\right)
$$

here $c_{2}(8)=3$ is the quadratic Casimir invariant of the octet and $c_{2}(3)=\frac{4}{3}$ is the quadratic Casimir invariant of the triplet. Saturating with low-lying resonances as in eq. (5.9) we have $\Delta^{2} \simeq 2(\ln 2) M_{\mathrm{V}}^{2}$, where $M_{\mathrm{V}}$ is the mass of the lightest hypervector resonance. Since four $\mathrm{G}_{\mathrm{H}}$ representations contribute to the $\mathrm{W}, \mathrm{Z}$ mass in this model, we expect $F \simeq 250 / \sqrt{4} \mathrm{GeV}=125 \mathrm{GeV}$, and therefore $M_{\mathrm{V}} \simeq\left(F / f_{\pi}\right) m_{\rho}=$ $1.0 \mathrm{TeV}$. Hence we estimate

$$
\begin{aligned}
& m_{8}^{2} \simeq \frac{9(\ln 2)}{2 \pi} \alpha_{c} M_{\mathrm{V}}^{2} \simeq(315 \mathrm{GeV})^{2}, \\
& m_{3}^{2} \simeq \frac{4}{9} m_{8}^{2} \simeq(210 \mathrm{GeV})^{2},
\end{aligned}
$$

taking $\alpha_{\mathrm{c}} \simeq 0.1$ at $1 \mathrm{TeV}$.

The degeneracy of the SU(2) triplets and singlets found in lowest order is accidental, and will be removed in higher order in $\alpha_{c}$. The $(8,1)$, for example, can 
annihilate into gluons, while the $(8,3)$ cannot, and it is presumably pushed up in mass. The splitting is of order $\left(1+\alpha_{c}\right)^{1 / 2}$, or about $5 \%$.

The electroweak splittings in the $(8,3)$ are second-order weak, as in example (d). Therefore, these splittings are tiny, being of order

$$
\frac{m_{8}^{+}-m_{8}^{0}}{m_{8}} \simeq \frac{1}{2} \frac{\Delta m_{8}^{2}}{m_{8}^{2}} \simeq \frac{1}{2} \frac{7^{2}}{300^{2}} \simeq 3 \times 10^{-4} .
$$

The splitting is about $70-100 \mathrm{MeV}$.

The electroweak splittings in the $(3,3)$ are first-order weak. Applying eq. (5.22) we find

$$
\begin{aligned}
\Delta m_{3}^{2}(\bar{E} U) & =\frac{3 g^{\prime 2}}{16 \pi^{2}}\left(\frac{10}{9}\right) \Delta^{2}=\frac{5 \alpha}{6 \alpha_{\mathrm{c}} \cos ^{2} \theta_{\mathrm{W}}} m_{3}^{2}, \\
\Delta m_{3}^{2}\left[\sqrt{\frac{1}{2}}(\bar{N} U-\bar{E} D)\right] & =\frac{3 g^{\prime 2}}{16 \pi^{2}}\left(\frac{4}{9}\right) \Delta^{2}=\frac{\alpha}{3 \alpha_{\mathrm{c}} \cos ^{2} \theta_{\mathrm{W}}} m_{3}^{2}, \\
\Delta m_{3}^{2}(\bar{N} D) & =-\frac{3 g^{\prime 2}}{16 \pi^{2}}\left(\frac{2}{9}\right) \Delta^{2}=-\frac{\alpha}{6 \alpha_{\mathrm{c}} \cos ^{2} \theta_{\mathrm{W}}} m_{3}^{2} .
\end{aligned}
$$

Numerically, we have

$$
m_{3}\left(Q=\frac{5}{3}\right)-m_{3}\left(Q=\frac{2}{3}\right) \simeq m_{3}\left(Q=\frac{2}{3}\right)-m_{3}\left(Q=-\frac{1}{3}\right) \simeq 5 \mathrm{GeV}
$$

$Q$ is the PGB electric charge.

It is clear that, if there really is a hypercolor interaction, we will have plenty of interesting spectroscopy to study. Of course, all the PGB's will receive mass from the sideways interaction as well, but the sideways contribution is small compared to the color and first-order weak contribution [7]. Electroweak splittings among the color-triplet PGB's of order a few GeV are observable in principle, because these states decay by sideways exchange, and are quite narrow [26].

(h) CHIRAL COLOR

Because we know that the electroweak group $S U(2) \times U(1)$ is broken when hypercolor gets strong, it is tempting to speculate that color SU(3) is embedded in a larger group which also breaks at a few hundred $\mathrm{GeV}$. For example, we have no evidence at present against the possibility that a chiral color group $S U(3)_{L} \times S U(3)_{R}$ is broken down to $\mathrm{SU}(3)_{\mathrm{c}}$ by the hypercolor interaction. One very interesting consequence of the chiral color is that it allows the colored PGB's to be considerably lighter than we calculated above.

Consider example (g) again, but now suppose that the left-handed hyperquarks are $(3,1)$ 's and the right-handed hyperquarks are $(1,3)$ 's under chiral color. 
[SU(3) $)_{L} \times S U(3)_{R}$ anomalies can be cancelled by additional fermions.] The breaking of $\mathrm{SU}(8)_{L} \times \mathrm{SU}(8)_{R}$ chiral symmetry to $\mathrm{SU}(8)_{V}$ forces $\mathrm{SU}(3)_{L} \times \mathrm{SU}(3)_{R}$ to break to the vector subgroup. If the chiral color gauge couplings are $g_{L}=g_{c} / \sin \theta$ and $g_{R}=g_{c} / \cos \theta$, where $\theta$ is a chiral color mixing angle, then the unbroken color currents are

$$
J^{a}=g_{\mathrm{c}}\left(J_{\mathrm{L}}^{a}+J_{\mathrm{R}}^{a}\right)
$$

and the massive chiral gluons couple to the currents

$$
J^{\prime a}=g_{\mathrm{c}}\left(J_{\mathrm{L}}^{a} \cot \theta-J_{\mathrm{R}}^{a} \tan \theta\right) .
$$

Here $J_{L, R}^{a}$ are the chiral color currents, normalized as in eq. (5.11).

In lowest order the vector gluons and broken chiral gluons give equal and opposite contributions to PGB masses. (This is just like example (d). Purely left-handed or purely right-handed interactions cannot contribute to the effective potential in lowest order.) But there is a non-vanishing contribution in the next order. Estimating the higher-order corrections as in example (d), we find that the octet and triplet PGB's receive masses

$$
\begin{aligned}
& m_{8}^{2}=\frac{9}{4 \pi} \alpha_{\mathrm{c}} \mu^{2} \ln \left(\frac{M_{\mathrm{V}}^{2}}{2 \mu^{2}}\right), \\
& m_{3}^{2}=\frac{4}{9} m_{8}^{2} .
\end{aligned}
$$

Here $\mu$ is the mass of a chiral gluon; since the Goldstone bosons couple with strength $F$ to the axial currents, we see from eq. (6.46) that $\mu^{2}=\frac{1}{4} g_{c}^{2} F^{2}(\cot \theta+$ $\tan \theta)^{2}=g_{c}^{2} F^{2} / \sin ^{2} 2 \theta$, and therefore

$$
\begin{aligned}
& m_{8}^{2} \simeq \frac{9 \alpha_{\mathrm{c}}^{2} F^{2}}{\sin ^{2} 2 \theta} \ln \left(\frac{M_{\mathrm{V}}^{2}}{2 \mu^{2}}\right) \simeq \frac{(68 \mathrm{GeV})^{2}}{\sin ^{2} 2 \theta}, \\
& m_{3}^{2} \simeq(45 \mathrm{GeV})^{2} / \sin ^{2} 2 \theta,
\end{aligned}
$$

taking $F=125 \mathrm{GeV}$. For the charge $\frac{5}{3}$ color triplet, the electroweak mass in eq. (6.43), $m_{3}^{2} \simeq(60 \mathrm{GeV})^{2}$, may actually be larger than the color contribution.

If chiral color exists, colored PGB's should be experimentally accessible fairly soon. Dimopoulos [26] has pointed out that the color octets can be singly produced, probably most easily in gluon-gluon collisions. The decays of the neutral ones sometimes give rise to exotic events in which a hard photon balances the momentum of a gluon jet. The color triplets can be pair-produced in $\mathrm{e}^{+} \mathrm{e}^{-}$colliding beam experiments. They decay into distinctive quark-lepton states [26]. 
We have assumed in this paper that the hypercolor group $G_{H}$ remains unbroken when hypercolor gets strong, but we cannot exclude the possibility that $G_{H}$ is actually embedded in a larger group which "self-destructs". In addition to the $\mathrm{W}^{ \pm}$, $Z$, and chiral gluons, there may be heavy hypergluons with masses of several hundred $\mathrm{GeV}$.

We have shown that the subgroup alignment problem can be explicitly solved in many hypercolor models, provided the unbroken isospin group $S_{f}$ is known. The physical consequences of subgroup alignment highlighted by our examples are diverse, and in some cases rather surprising.

We hope that our analysis of subgroup alignment will provide the serious prospective model-builder with a bit of guidance. We can, at least, exclude models in which the electroweak gauge group fails to break down as desired.

We have stressed the impact of the alignment of subgroups on the breakdown of the electroweak gauge group, but we wish to conclude by pointing out another context in which an alignment problem must be solved. In a realistic theory, a sideways interaction [6-8] is required to generate the masses of quarks and leptons. We must account for the dynamical breakdown of the sideways gauge group. One possibility is that the sideways group is broken by another new strong interaction [6], just as the electroweak group is broken by the hypercolor interaction. In that case, the sideways gauge group can be treated as a weakly gauged subgroup of an approximate flavor-symmetry group. Hence, the general formalism developed in this paper can be employed to determine the pattern of the sideways breakdown.

I thank Michael Peskin for informing me about his recent work, and for helpful comments. He has independently obtained many of the results reported here. I am grateful to Kenneth Lane for suggesting that spectral-function sum rules can be used to solve the subgroup alignment problem, and to Estia Eichten for many illuminating conversations while this work was in progress. I also thank Steven Weinberg and Howard Georgi for advice and encouragement.

\section{References}

[1] S. Weinberg, Phys. Rev. Lett. 19 (1967) 1264;

A. Salam, in Elementary particle theory, ed. N. Svartholm, (Almqvist and Wiksell, Stockholm, 1968)

[2] S. Weinberg, Phys. Lett. 82B (1979) 387

[3] S. Weinberg, Phys. Rev. D13 (1976) 974; D19 (1979) 1277

[4] L. Susskind, Phys. Rev. D20 (1979) 2619

[5] H. Georgi and S.L. Glashow, Phys. Rev. Lett. 32 (1974) 438;

H. Georgi, H.R. Quinn and S. Weinberg, Phys. Rev. Lett. 33 (1974) 451

[6] S. Dimopoulos and L. Susskind, Nucl. Phys. B155 (1979) 237

[7] E. Eichten and K. Lane, Phys. Lett. 90B (1980) 125

[8] S. Weinberg, unpublished

[9] R. Dashen, Phys. Rev. D3 (1971) 1879

[10] E. Eichten, K. Lane and J. Preskill, Phys. Rev. Lett. 45 (1980) 225 
[11] E. Eichten, K. Lane and J. Preskill, in preparation

[12] J. Preskill, Harvard Ph.D. thesis (1980), unpublished

[13] S. Dimopoulos and L. Susskind, A technicolored solution to the strong $C P$ problem, Columbia preprint 79-0196 (1979)

[14] M.E. Peskin, The alignment of the vacuum in theories of technicolor, Saclay preprint DPh-T/80/46 (1980)

[15] S. Weinberg, Phys. Rev. Lett. 18 (1967) 507

[16] H. Georgi, Nucl. Phys. B156 (1979) 126;

M. Peskin, in preparation;

S. Dimopoulos, S. Raby and L. Susskind, Tumbling gauge theories, Stanford preprint ITP-653 (1979)

[17] J.S. Bell and R. Jackiw, Nuovo Cim. 51 (1969) 47;

S.L. Adler, Phys. Rev. 1772426 (1969)

[18] Y. Nambu and G. Jona-Lasinio, Phys. Rev. 122 (1961) 345;

G. 't Hooft, Naturalness, chiral symmetry and spontaneous chiral symmetry breaking, Lectures at Cargése Summer Inst. (1979)

[19] L. Michel and L.A. Radicati, Ann. of Phys. 66 (1971) 758

[20] R. Dashen, Phys. Rev. 183 (1969) 1245

[21] J. Schwinger, Phys. Rev. 125 (1962) 397; 128 (1962) 2425

[22] K. Wilson, Phys. Rev. 179 (1969) 1499

[23] C. Bernard, A. Duncan, J. Lo Secco and S. Weinberg, Phys. Rev. D12 (1975) 792

[24] T. Das, G.S. Guralnik, V.S. Mathur, F.E. Low and J.E. Young, Phys. Rev. Lett. 18 (1967) 759

[25] J.J. Sakurai, Phys. Rev. Lett. 17 (1966) 552

[26] S. Dimopoulos, Nucl. Phys. B168 (1980) 69

[27] B. Holdom, A realistic model with dynamically broken symmetries, Harvard preprint HUTP$80 /$ A010 (1980)

[28] E. Farhi and L. Susskind, Phys. Rev. D20 (1979) 3404

[29] T. Hagiwara and R.N. Mohapatra, Phys. Rev. D11 (1975) 2223 